\title{
Adaptive Control of Peak Current Mode Controlled Boost Converter Supplied by Fuel Cell
}

\author{
Toni Bjažić†, Željko Ban*, and Nedjeljko Perić* \\ ${ }^{\dagger}$ Professional Study in Mechatronics, Polytechnic of Zagreb, Zagreb, Croatia \\ *Faculty of Electrical Engineering and Computing, University of Zagreb, Zagreb, Croatia
}

\begin{abstract}
Adaptive control of a peak current mode controlled (PCM) boost converter supplied by a PEM fuel cell is described in this paper. The adaptive controller with reference model and signal adaptation is developed in order to compensate the deviation of the response during the change of the operating point. The procedure for determining the adaptive algorithm's weighting coefficients, based on a combination of the pole-zero placement method and an optimization method is proposed. After applying the proposed procedure, the optimal adaptive algorithm's weighting coefficients can be determined in just a few iterations, without the use of a computer, thus greatly facilitating the application of the algorithm in real systems. Simulation and experimental results show that the dynamic behavior of a highly nonlinear control system with a fuel cell and a PCM boost converter, can fairly accurately be described by the dynamic behavior of the reference model, i.e., a linear system with constant parameters.
\end{abstract}

Key words: Adaptive control, reduced order reference model, signal adaptation, boost converter, peak current mode control, PEM fuel cell

\section{INTRODUCTION}

Renewable energy sources are becoming more and more important in the global electricity network. Particularly, fuel cells have a great potential for replacing fossil fuel based energy sources, as a result of their availability and especially cost reduction due to technology. DC/DC converters, which must ensure a stable voltage link (e.g. microgrid voltage) irrespective of the operating point determined by the load connected to the link, are indispensable parts of systems with fuel cells. Thereby, different topologies have been researched [1]-[11].

It is shown that some advanced control techniques should be used to control the voltage of systems with fuel cells. Some of these methods include indirect model reference adaptive control [12], model reference adaptive control with signal adaptation algorithm [13], and nonlinear control [14]-[20]. However, the dynamics of such systems is always slow compared to the dynamics of DC/DC converters.

This paper deals with a concept of peak current mode controlled (PCM) boost converter supplied by a PEM fuel

Manuscript received Sep. 1, 2012; revised Nov. 16, 2012

Recommended for publication by Associate Editor Se-Kyo Chung.

${ }^{\dagger}$ Corresponding Author: toni.bjazic@tvz.hr

Tel: +385989352762, Polytechnic of Zagreb

Faculty of Electrical Engineering and Computing, University of Zagreb, Croatia cell. The output voltage of the PEM fuel cell BCS 64-32 [22] is in range from $19 \mathrm{~V}$ to $31 \mathrm{~V}$ and the boost converter has to raise it to $50 \mathrm{~V}$. This system is part of a microgrid in the Laboratory for Renewable Energy Systems (LARES) [23], built at the University of Zagreb, Faculty of Electrical Engineering and Computing.

The system description and the procedure of modeling the system with PCM boost converter supplied by a fuel cell suitable for controller design purposes is presented in detail in [24]. The introduction given in [24] also applies in this paper. Since the system with the PCM boost converter supplied by the PEM fuel cell is a highly nonlinear and non-minimum phase system, the classical control approach could not successfully deal with control problem. The influence of the non-minimum phase behavior to system response can be reduced by a careful design of the boost converter components, as shown in [24], and nonlinearities are proposed to be compensated for by an adaptive controller using the reference model and signal adaptation algorithm of a reduced order as described in this paper.

This paper is divided into several sections. Inner control loop of the adaptive system, implemented using a basic PI controller as an auxiliary component of the adaptive system, is described in Section II.

A model reference adaptive control scheme with signal adaptation algorithm is described in Section III. A full order 
algorithm and a reduced order algorithm are described in detail, and the procedure of model order reduction applied to the PCM boost converter supplied by the PEM fuel cell is given.

An application of the model reference adaptive control with reduced order signal adaptation algorithm to a process with the PCM boost converter supplied by the PEM fuel cell is described in Section IV. Likewise, a procedure for the determination of optimal adaptive algorithm coefficients is described, and simulation and experimental results are given.

Finally, important conclusions are given in Section V.

\section{ADAPTIVE SYSTEM INNER CONTROL LOOP DESIGN}

The control system installed in the Laboratory for Renewable Energy Systems (LARES) [23], [24], consists of a $500 \mathrm{~W}$ fuel cell BCS 64-32 (Fig. 1), manufactured by BCS Fuel Cells Inc., a $450 \mathrm{~W}$ boost converter specifically designed and built in the Croatian company Mareton Power Electronics (Fig. 2), a Magna Power Electronics fuel cell emulator (Fig. 3) [25], [26], and finally a Compact RIO (cRIO) 9024 digital controller with input-output modules, manufactured by the National Instruments company, which will be used for the experimental identification of the system parameters, estimation of state variables and implementation of digital control algorithms [24], [27], [28].

Inner control loop of the adaptive system is implemented with a basic PI controller (Fig. 4). The basic PI controller, as an auxiliary component of the adaptive control system, is designed for the nominal operating point of the process, determined by the converter output current $I_{\text {out }}=9 \mathrm{~A}$. In all other operating points the control system with the basic PI controller must be stable. Additional required conditions include good and fast compensation of disturbances (change of output current or resistive load at converter output), and minimum oscillations.

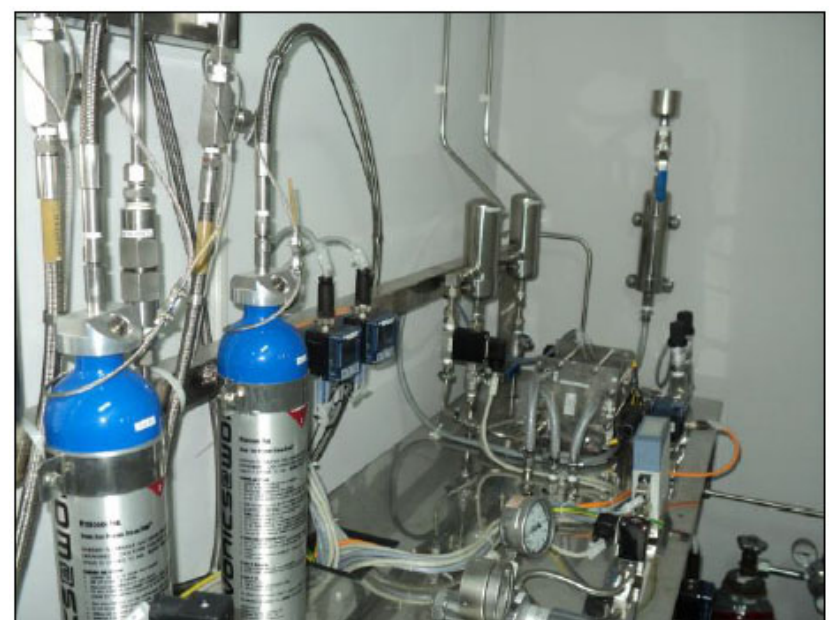

Fig. 1. The part of the hydrogen facility in the Laboratory for Renewable Energy Systems - LARES.

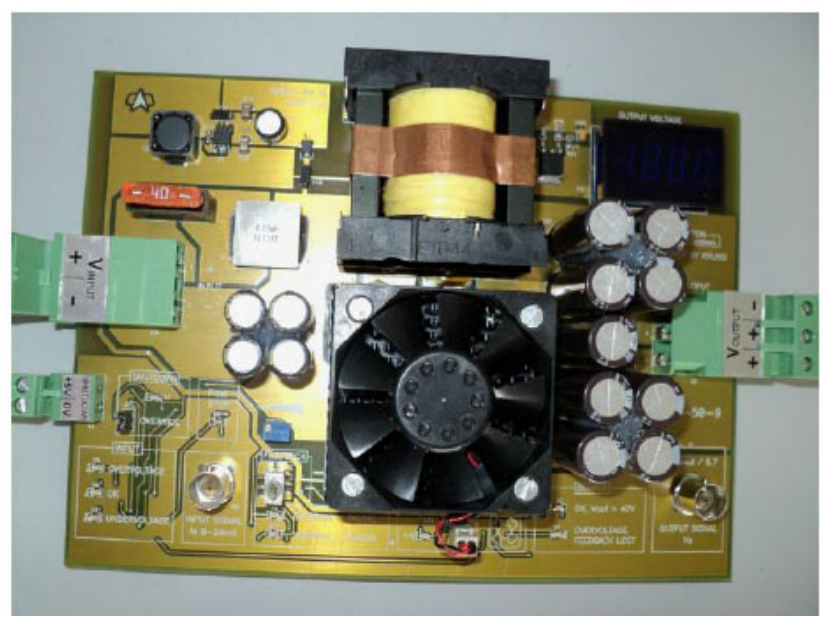

Fig. 2. DC-DC boost converter, designed in Mareton Power Electronics.

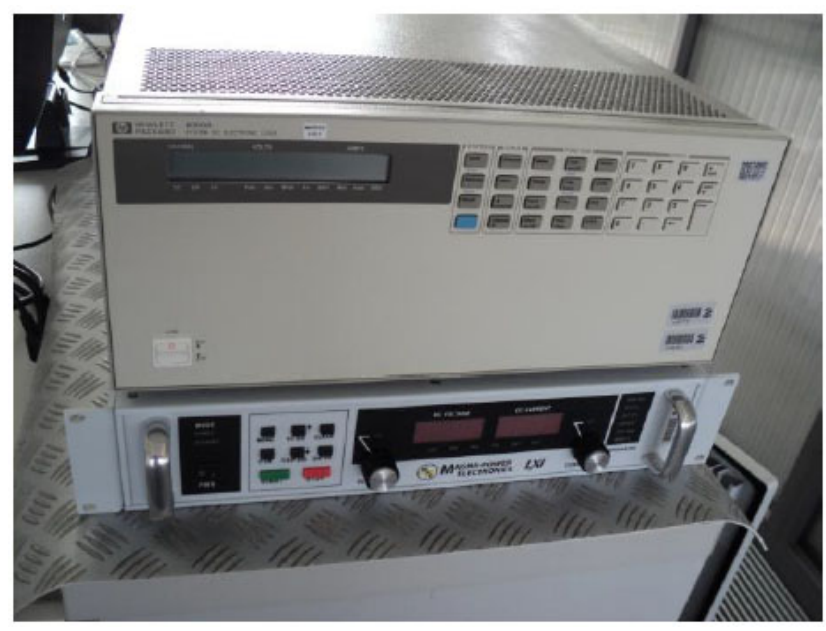

Fig. 3. Front panels of the fuel cell emulator Magna Power Electronics and the programmable electronic load HP 6050A.

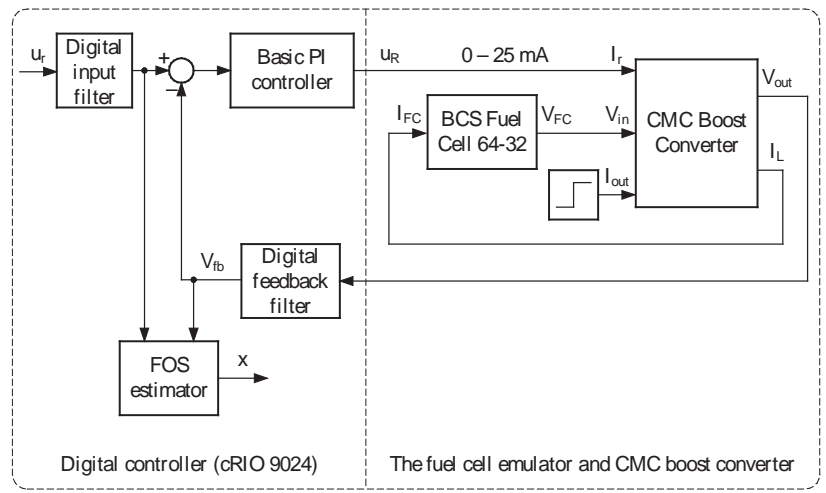

Fig. 4. The block schematics of the control system with the basic PI controller and FOS estimator.

A continuous transfer function of the PI controller is:

$$
G_{R}(s)=\frac{\Delta i_{r}(s)}{\Delta v_{\text {out }, r}(s)-\Delta v_{f b}(s)}=K_{R} \cdot \frac{1+T_{I} s}{T_{I} s},
$$


where:

$\Delta i_{r}(s)$ - controller output signal (boost converter control signal) $(0-25 \mathrm{~mA})$,

$\Delta v_{\text {out }, r}(s)$ - output voltage reference value (setpoint)

(V),

$\Delta v_{f b}(s)$ - measured output voltage of the boost converter (feedback voltage) (V),

$K_{R}$ - controller gain and

$T_{I}$ - controller integral time constant (s).

Designations $\Delta$ in (1) and further in paper represent changes of values around the operating point.

The process in its nominal operating point is described by [24]:

$$
G_{p}(s)=\frac{\Delta v_{f b}(s)}{\Delta i_{r}(s)}=\frac{K_{p} \cdot\left(1+T_{D p} s\right)}{\left(1+T_{1} s\right)\left(1+T_{2} s\right)\left(1+T_{f b} s\right)}
$$

where: $K_{p}=461, T_{D p}=0.1576 \mathrm{~s}, \quad T_{1}=0.1142 \mathrm{~s}$, $T_{2}=0.0171 \mathrm{~s}, T_{f b}=0.00035 \mathrm{~s}$.

All requirements on the basic PI controller are satisfied by the choice of its parameters $K_{R}=0.085$ and $T_{I}=4.4 \mathrm{~ms}$ [21].

\section{MODEL REFERENCE ADAPTIVE CONTROL WITH SIGNAL ADAPTATION ALGORITHM}

The influence of small changes of parameters on system behavior can be sufficiently well compensated for by the classical control algorithms (PID). However, significant changes in parameters cause large deviations from the desired behavior of the control system. To achieve the desired behavior of such a control system, it is necessary to apply some of the advanced control algorithms.

The compensation of changes of the system parameters or system nonlinearities can be achieved by gain scheduling controllers [29], [30] or their advanced forms such as linear parameter varying (LPV) control methods [31] and multimodel approach [32], then by selftuning controllers [29] or by model reference adaptive control (MRAC) [29], [30], [33], [34]. System nonlinearities can be compensated for by model predictive control (MPC) as well [35], [36].

The gain scheduling controllers are suitable if there exists a connection between measurable process variables and process parameters. By sensing the process variables and applying the connection between them and the process parameters, the controller parameters are changed to compensate for the effects of changes of process parameters.

The selftuning controller usually requires persistent excitation for the process identification part of the algorithm, which is forbidden in some processes.

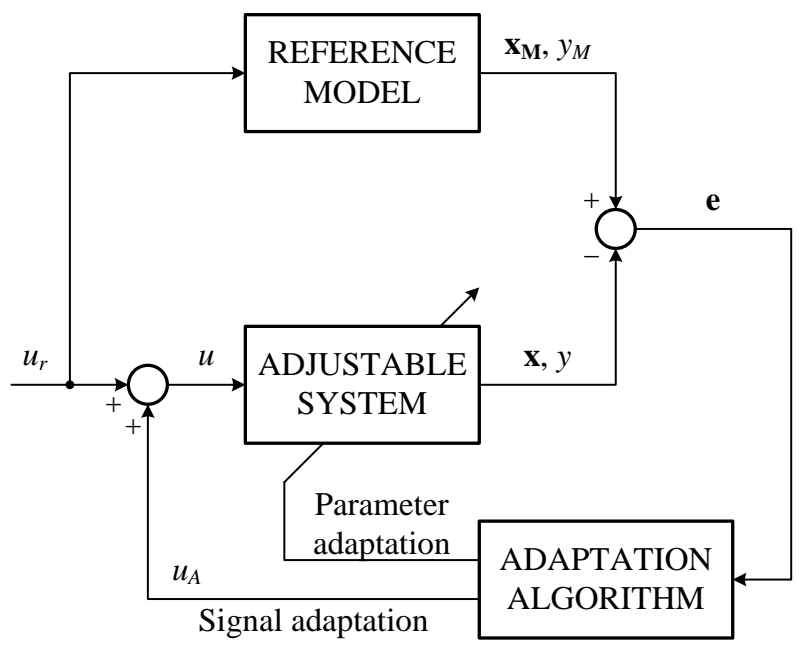

Fig. 5. The block schematics of the model reference adaptive system.

The model reference adaptive controllers (Fig. 5) have the advantage of simplicity of implementation and at the same time speed of adaptation, because they do not require continuous estimation of process parameters. The basic task of the model reference adaptive system is to achieve that an output signal of the process asymptotically follows the output signal of the reference model. Model following is ensured by an adaptation mechanism which adjusts the parameters of the adjustable system (parameter adaptation) or generates an additional control (adaptation) signal which minimizes the difference between output signals or state variables of the adjustable system and output signals or state variables of the reference model (signal adaptation).

Parameter adaptation with a reference model contains integral parts, thus requiring more iterations for adjusting the optimal controller parameters and a new adjustment every time when process parameters are changed [37]. The advantage of signal adaptation with a reference model is that it does not contain any integral parts and therefore does not require learning. It acts instantly (in the first iteration) on any deviation between output signals or state variables of the adjustable system and the reference model.

Conventional approach in the adaptive control theory requires the full order of the reference model as well as full state variable vector in the adaptation mechanism. However, usage of the reduced order of the reference model and adaptation algorithm could simplify the controller and keep the satisfactory response.

The conventional adaptive control theory with full order reference model is used for developing the structure of the control algorithm, and after that the algorithm is modified in order to become more suitable for control of the described converter supplied by the fuel cell. 


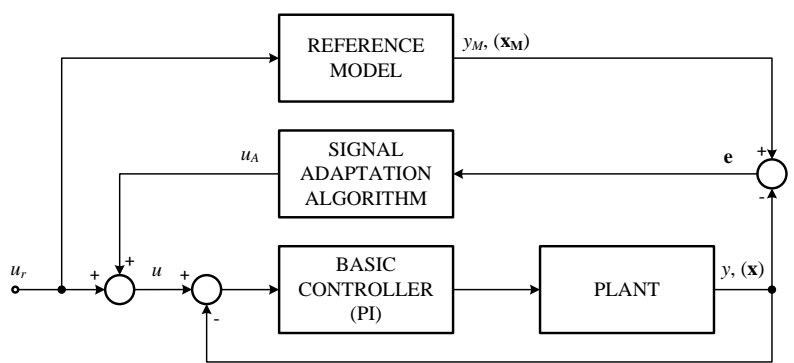

Fig. 6. The adaptive system with the reference model and the signal adaptation algorithm in the outer control loop.

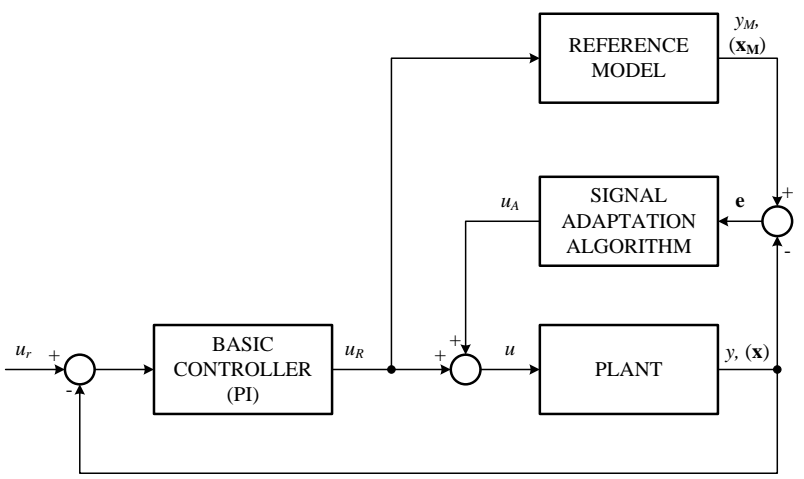

Fig. 7. The adaptive system with the reference model and the signal adaptation algorithm in the inner control loop.

\section{A. Signal adaptation algorithm with a full order reference model}

The signal adaptation algorithm generates an additional control signal $u_{A}$, which minimizes the difference between reference model output $y_{M}$ and adjustable system $y$ (Figs. 6 and 7). The adaptation signal acts at the system input so that the adaptation mechanism forms an outer control loop, while the adjustable system with the basic controller forms an inner control loop (Fig. 6).

The second possibility is that the adaptation signal acts after the basic controller (Fig. 7), so that the adaptation mechanism forms an inner control loop, while the basic controller acts in the outer loop.

Single input single output (SISO) linear time invariant systems can be described in state space form:

$$
\dot{\mathbf{x}}(t)=\mathbf{A x}(t)+\mathbf{b} u(t),
$$

where:

$$
\begin{aligned}
& \text { A - system matrix }(n \times n), \\
& \mathbf{b} \text { - system input vector }(n \times 1), \\
& \mathbf{x} \text { - vector of system state space variables }(n \times 1) \text {, } \\
& u \text { - system input }(1 \times 1) .
\end{aligned}
$$

The reference model is described by:

$$
\dot{\mathbf{x}}_{\mathbf{M}}(t)=\mathbf{A}_{\mathbf{M}} \mathbf{x}_{\mathbf{M}}(t)+\mathbf{b}_{\mathbf{M}} u_{x}(t)
$$

where:

$\mathbf{A}_{\mathbf{M}}$ - reference model matrix $(n \times n)$,

$\mathbf{b}_{\mathbf{M}}$ - reference model input vector $(n \times 1)$,

$\mathbf{x}_{\mathbf{M}}$ - vector of reference model state space variables $(n \times 1)$,

$u_{x}$ - referent signal $u_{r}$ or $u_{R}(1 \times 1)$, depending on

adaptation structure (Fig. 6 or 7).

According to Figs. 6 and 7, signal $u_{x}$ is determined by:

$$
u_{x}(t)=u(t)-u_{A}(t)
$$

where: $u_{A}$ - adaptation signal.

The error following vector is determined by:

$$
\mathbf{e}(t)=\mathbf{x}_{\mathbf{M}}(t)-\mathbf{x}(t) \text {. }
$$

From the state space representation of system (3) and reference model (4), equations (5) and (6), and after adding and subtracting $\mathbf{A}_{\mathbf{M}} \mathbf{x}$, the expression for the error derivative is obtained:

$$
\dot{\mathbf{e}}(t)=\dot{\mathbf{x}}_{\mathbf{M}}(t)-\dot{\mathbf{x}}(t)=\mathbf{A}_{\mathbf{M}} \mathbf{e}(t)+\boldsymbol{\sigma}(t)-\mathbf{b} u_{A}(t),
$$

where:

$$
\boldsymbol{\sigma}(t)=\left(\mathbf{A}_{\mathbf{M}}-\mathbf{A}\right) \mathbf{x}(t)+\left(\mathbf{b}_{\mathbf{M}}-\mathbf{b}\right) u_{x}(t) .
$$

Vector $\boldsymbol{\sigma}$ is determined by variations of the system (plant) parameters from the reference model parameters.

The adaptation signal $u_{A}$, which will ensure the complete following of the reference model, is obtained by solving the equation (7) by $u_{A}$ and including the conditions for complete following of the reference model $(\mathbf{e}=\mathbf{0}$ and $\dot{\mathbf{e}}=\mathbf{0})$ :

$$
u_{A}(t)=\mathbf{b}^{+} \sigma(\mathbf{x}, t)
$$

where: $\mathbf{b}^{+}=\left(\mathbf{b}^{T} \mathbf{b}\right)^{-1} \mathbf{b}^{T}-$ Moore-Penrose pseudoinverse of vector $\mathbf{b}$.

On substituting (9) into (7), the following is obtained:

$$
\dot{\mathbf{e}}(t)=\mathbf{A}_{\mathbf{M}} \mathbf{e}(t)+\left(\mathbf{I}-\mathbf{b b}^{+}\right) \sigma(\mathbf{x}, t)
$$

from which the necessary and sufficient Ercberger's condition for complete following of the reference model is obtained [38]:

$$
\left(\mathbf{I}-\mathbf{b b}^{+}\right) \boldsymbol{\sigma}(\mathbf{x}, t)=\mathbf{0},
$$

where: I - unity matrix.

Since the products in (11) are different from zero, to fulfill the Ercberger's condition (11), it is necessary to synthesize the adaptation signal $u_{A}$, which is contained in the vector $\boldsymbol{\sigma}$.

The stability of the adaptive controller can be shown using Lyapunov's stability criterion, provided that all state space variables of the system are known. The appropriate Lyapunov's positive definite function has a quadratic form:

$$
V=\frac{1}{2} \mathbf{e}^{T} \mathbf{P e},
$$

where: $\mathbf{P}$ - positive definite matrix determined by the well-known Lyapunov's matrix equation:

$$
\mathbf{A}^{T} \mathbf{P}+\mathbf{P A}=-\mathbf{Q},
$$

where: $\mathbf{Q}$ - positive definite matrix. 
The derivation of Lyapunov's function (12) is determined by:

$$
\dot{V}=\dot{\mathbf{e}}^{T} \mathbf{P e}+\mathbf{e}^{T} \mathbf{P} \dot{\mathbf{e}} .
$$

On substituting (7) into (14), the following is obtained:

$$
\dot{V}=-\mathbf{e}^{T} \mathbf{Q e}+2 \mathbf{e}^{T} \mathbf{P} \boldsymbol{\sigma}-2 \mathbf{e}^{T} \mathbf{P} \mathbf{b} u_{A} .
$$

The derivation of Lyapunov's function (15) will be negative definite if the sum of the second and the third operand in relation (15) is negative, i.e. if the following holds:

$$
\mathbf{e}^{T} \mathbf{P b} u_{A}>\mathbf{e}^{T} \mathbf{P} \sigma
$$

The product $\mathbf{e}^{T} \mathbf{P b}$ is scalar for SISO systems, so it can be written:

$$
\mathbf{e}^{T} \mathbf{P b}=\left(\mathbf{e}^{T} \mathbf{P} \mathbf{b}\right)^{T}=\mathbf{b}^{T} \mathbf{P}^{T} \mathbf{e}=\mathbf{b}^{T} \mathbf{P e} .
$$

The scalar (17) is called the generalized error and is designated as $v$ :

$$
v(t)=\mathbf{b}^{T} \mathbf{P e}(t)
$$

The part $\mathbf{b}^{T} \mathbf{P}$ of the expression (18) is called an error weighting coefficient vector and is designated as $\mathbf{d}$ :

$$
\mathbf{d}^{T}=\mathbf{b}^{T} \mathbf{P} \text {. }
$$

By dividing the inequality (16) with the scalar $\mathbf{e}^{T} \mathbf{P b}$, i.e. the generalized error, the following is obtained:

$$
\begin{aligned}
& u_{A}>\frac{\mathbf{e}^{T} \mathbf{P} \sigma}{\mathbf{e}^{T} \mathbf{P b}}, \quad \mathbf{e}^{T} \mathbf{P b}>0, \\
& u_{A}<\frac{\mathbf{e}^{T} \mathbf{P} \sigma}{\mathbf{e}^{T} \mathbf{P b}}, \quad \mathbf{e}^{T} \mathbf{P b}<0,
\end{aligned}
$$

or shorter:

$$
\begin{array}{ll}
u_{A}>\mathbf{b}^{+} \sigma, & \mathbf{e}^{T} \mathbf{P b}>0, \\
u_{A}<\mathbf{b}^{+} \sigma, & \mathbf{e}^{T} \mathbf{P} \mathbf{b}<0 .
\end{array}
$$

The conditions (20) or (21) will be satisfied if the adaptation algorithm has the form:

$$
u_{A}(t)=h \operatorname{sign}(v(t)) \text {, }
$$

where: $h$ - adaptation coefficient, which according to conditions (20) or (21), has to have a minimal value determined by:

$$
h>\left\|\mathbf{b}^{+}\right\| \cdot\|\boldsymbol{\sigma}\|
$$

For the positive definite Lyapunov's function $(V>0)$, whose derivative is negative definite $(\dot{V}<0)$, it is necessary and sufficient to fulfill the Ercberger's condition (11) in order to ensure complete following of the reference model.

To determine the minimum value of the adaptation coefficient $h$, it is necessary to estimate the matrix norms in (23). The norm estimate of the vector $\boldsymbol{\sigma}$ is obtained from (8):

$$
\|\boldsymbol{\sigma}\| \leq\left\|\mathbf{A}_{\mathbf{M}}-\mathbf{A}\right\| \cdot\|\mathbf{x}\|+\left\|\mathbf{b}_{\mathbf{M}}-\mathbf{b}\right\| \cdot\left\|u_{x}\right\| \cdot
$$

For estimating the norms in (24), matrices $\mathbf{A}_{\mathbf{M}}$ and $\mathbf{b}_{\mathbf{M}}$, which are known and have constant coefficients are used, while matrices $\mathbf{A}$ and $\mathbf{b}$ are used for the case of maximum deviation of adjustable system parameters from the reference model parameters. The norms $\|\mathbf{x}\|$ and $\left\|u_{x}\right\|$ can be estimated by simulation of the adaptive system for the previously determined parameters of the adjustable system and for the highest allowed change of the system input signal.

Since the signal adaptation algorithm (22) causes constant high frequency oscillations in the system, which is not suitable for most real systems, modification of the algorithm is needed. One of the possible modifications of the adaptation algorithm (22), which removes the constant high frequency oscillations in the system, is replacing the sign function in the algorithm by a saturation function:

$$
\begin{gathered}
u_{A}(t)=\operatorname{sat}(v(t), h)=\left\{\begin{array}{ccc}
h, & \text { for } & v(t)>v_{s} \\
K_{v} v(t), & \text { for } & |v(t)| \leq v_{s}, \\
-h, & \text { for } & v(t)<-v_{s}
\end{array}\right. \\
v(t)=\mathbf{d}^{T} \mathbf{e}(t), \quad \mathbf{d}^{T}=\mathbf{b}^{T} \mathbf{P},
\end{gathered}
$$

where:

$$
\begin{aligned}
& K_{v} \text { - generalized error gain coefficient, } \\
& v_{s} \text { - linear area of the saturation function. }
\end{aligned}
$$

If using the signal adaptation algorithm with the saturation function (25), the adaptive system is completely linear in a vicinity of an equilibrium point, i.e. the adaptation signal can be expressed as:

$$
u_{A}=K_{v} \mathbf{d}^{T} \mathbf{e} .
$$

The differential equation of the error in that case has the form:

$$
\dot{\mathbf{e}}=\left(\mathbf{A}_{\mathbf{M}}-K_{\nu} \mathbf{b}_{\mathbf{M}} \mathbf{d}^{T}\right) \mathbf{e}+\left(\mathbf{A}_{\mathbf{M}}-\mathbf{A}\right) \mathbf{x}+\left(\mathbf{b}_{\mathbf{M}}-\mathbf{b}\right) u_{x},
$$

and the stability condition is that the matrix $\left(\mathbf{A}_{\mathbf{M}}-K_{\nu} \mathbf{b}_{\mathbf{M}} \mathbf{d}^{T}\right)$ in (28) is Hurwitz's matrix, i.e. its eigenvalues (characteristic values) must lie in the left half-plane of the complex s-plane.

Beyond the linear area of the saturation function, the adaptation algorithm (25) behaves like the adaptation algorithm with the sign function (22), thus ensuring that the generalized error will return to the linear area, i.e. ensures the stability of the adaptive control system.

As may be seen from the differential equation of the error (28), complete following of the reference model is not possible, due to the last two uncompensated addends.

The coefficients of matrix $\mathbf{P}$, and thereby of vector $\mathbf{d}^{T}$ can be determined from Lyapunov's matrix equation (13), with given coefficients of matrix $\mathbf{Q}$. However, due to arbitrary choice of the coefficients of matrix $\mathbf{Q}$, the error weighting coefficients obtained in that way do not result in best adaptation, i.e. the smallest error value in the transient response, so these weighting coefficients are not optimal. To obtain the best (optimal) adaptation system, all coefficients of matrix $\mathbf{Q}$ should be optimized. 


\section{B. Signal adaptation algorithm with a reduced order reference model}

The major disadvantage of the previously described signal adaptation algorithm with the full order reference model is that a relatively large number of adaptive algorithm coefficients has to be determined (at least equal to the system order $n$ ). Since the procedure of determining the optimal coefficients is neither straightforward nor simple due to the inevitable use of some sort of optimization, it is prudent to reduce the number of coefficients in the algorithm, and at the same time retain the good properties of adaptation. In this regard, the adjustable system and reference model are described with mathematical models of reduced order.

Real processes can be very well approximated by a model of third order or lower in an operating point. By carefully selecting the parameters of the model, the maximum approximation error can be reduced to within $10 \%$, and can be merged with the rest of the process uncertainties, which are always present, because we always make some modeling assumptions that affect the accuracy of modeling to a greater or lesser degree.

In that case, the systems with one input and one output (SISO) in the operating point can be described in state space form by linear time invariant terms and unknown but bounded function of uncertainties and disturbances, with $n \leq 3$ :

$$
\dot{\mathbf{x}}(t)=\mathbf{A x}(t)+\mathbf{b} u(t)+\mathbf{f}(t, \mathbf{x}, u)
$$

where:

$$
\begin{aligned}
& \text { A - system matrix ( }(n \times n, n \leq 3), \\
& \mathbf{b} \text { - system input vector }(n \times 1, n \leq 3), \\
& \mathbf{x} \text { - vector of system state space variables }(n \times 1, n \leq 3), \\
& u \text { - system input }(1 \times 1) \text {, } \\
& \mathbf{f}(t, \mathbf{x}, u) \text { - unknown, but bounded function of } \\
& \quad \text { uncertainties and disturbances. }
\end{aligned}
$$

The reference model is described by (4), and it has to be of the same order as the reduced system (29).

From the state space descriptions of the reduced system (29) and reference model (4), expressions (5) and (6), and after adding and subtracting the term $\mathbf{A}_{\mathbf{M}} \mathbf{x}$, an expression for the error derivative of the reduced order adaptive system is obtained:

$$
\dot{\mathbf{e}}(t)=\dot{\mathbf{x}}_{\mathbf{M}}(t)-\dot{\mathbf{x}}(t)=\mathbf{A}_{\mathbf{M}} \mathbf{e}(t)+\boldsymbol{\sigma}(t)-\mathbf{b} u_{A}(t),
$$

where:

$$
\boldsymbol{\sigma}(t)=\left(\mathbf{A}_{\mathbf{M}}-\mathbf{A}\right) \mathbf{x}(t)+\left(\mathbf{b}_{\mathbf{M}}-\mathbf{b}\right) u_{x}(t)-\mathbf{f}(t, \mathbf{x}, u) .
$$

The adaptation signal $u_{A}$, which ensures the complete following of the reference model, is obtained in the same way as in the case of using the full order state variables vector (expressions (9) - (15)) and it is determined by (22). The only difference is in the estimate of the minimal value of the adaptation coefficient $h$ :

$$
h \geq\left\|\mathbf{b}^{+}\right\| \cdot\|\boldsymbol{\sigma}\|,
$$

where the norm of the vector $\boldsymbol{\sigma}$ is estimated by:

$$
\|\sigma\| \leq\left\|\mathbf{A}_{\mathbf{M}}-\mathbf{A}\right\| \cdot\|\mathbf{x}\|+\left\|\mathbf{b}_{\mathbf{M}}-\mathbf{b}\right\| \cdot\left\|u_{x}\right\|+\|\mathbf{f}(t, \mathbf{x}, u)\| .
$$

For estimating the norms in (33), matrices $\mathbf{A}_{\mathbf{M}}$ and $\mathbf{b}_{\mathbf{M}}$, which have known and constant coefficients, are used, while matrices $\mathbf{A}$ and $\mathbf{b}$ are taken for the case of maximum deviation of the adjustable system parameters from the reference model parameters. The norms $\|\mathbf{x}\|$ and $\left\|u_{x}\right\|$ can be estimated by simulation of the adaptive system for the previously determined parameters of the adjustable system and for the highest allowed change of the system input signal. The norm $\|\mathbf{f}(t, \mathbf{x}, u)\|$ is determined from the maximum error that is consciously done by approximation of the real system with the reduced order model.

If using the signal adaptation algorithm with the saturation function (25) for eliminating constant high frequency oscillations, the differential equation of the error has the form:

$$
\begin{aligned}
\dot{\mathbf{e}}=( & \left(\mathbf{A}_{\mathrm{M}}-K_{v} \mathbf{b}_{\mathrm{M}} \mathbf{d}^{T}\right) \mathbf{e}+\left(\mathbf{A}_{\mathrm{M}}-\mathbf{A}\right) \mathbf{x}+ \\
& +\left(\mathbf{b}_{\mathrm{M}}-\mathbf{b}\right) u_{x}-\mathbf{f}(t, \mathbf{x}, u),
\end{aligned}
$$

and the stability condition is the same as in the case of using the adaptive algorithm of the full order, i.e. the matrix $\left(\mathbf{A}_{\mathrm{M}}-K_{v} \mathbf{b}_{\mathrm{M}} \mathbf{d}^{T}\right)$ in (34) has to be a Hurwitz matrix.

As may be seen from the differential equation of the error (34) complete following of the reference model is not possible, due to uncompensated second and third addend and unknown function of uncertainties and disturbances $\mathbf{f}$. As a result, the coefficients of the adaptive algorithm have to be chosen so that the maximum error in the transient response of the system is kept as small as possible.

\section{Adjustable system and reference model order reduction}

The process which consists of the PCM boost converter and the fuel cell emulator is described in the nominal operating point by the transfer function (2), while the basic controller, whose parameters are constant independently of the operating point $\left(K_{R}=0.085, T_{I}=4.4 \mathrm{~ms}\right)$, is described by the transfer function (1). Therefore, the closed loop transfer function of the control system with the basic controller in the nominal operating point is given by:

$$
G_{c l}(s)=\frac{\Delta v_{f b}(s)}{\Delta u_{r}(s)}=\frac{G_{R}(s) G_{p}(s)}{1+G_{R}(s) G_{p}(s)},
$$

where: $\Delta u_{r}$ - the change of referent signal of the control system.

It is obvious that the transfer function $G_{c l}(s)$ is of the fourth order. A pole-zero map of the transfer function $G_{c l}(s)$ is shown in Fig. 8. 


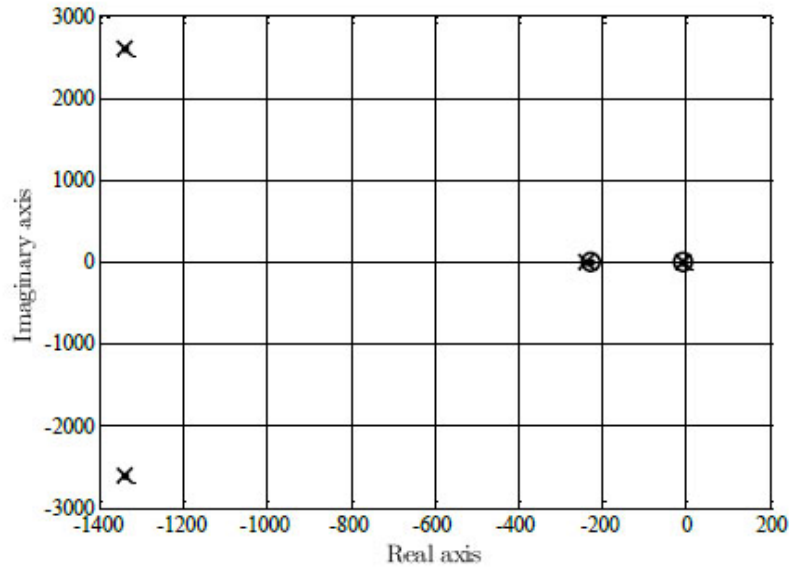

Fig. 8. The pole-zero map of the transfer function $G_{c l}(s)$ (35).

It is clearly seen from Fig. 8 that two pairs of poles-zeros are placed very close to each other, and can be canceled. The error that is made by cancellation can be partially compensated for by the correction of the placement of the remaining pair of poles.

Therefore, the transfer function of the reduced system (without the filter at the referent signal branch) is obtained:

$$
G_{c l, r}(s)=\frac{\Delta v_{f b}(s)}{\Delta u_{r}(s)}=\frac{\omega_{0}^{2}}{s^{2}+2 \zeta \omega_{0} s+\omega_{0}^{2}},
$$

where the relative damping coefficient $\zeta$ and natural frequency of undamped oscillations $\omega_{0}$ are determined by minimizing the difference between the experimentally recorded step response of the feedback voltage and the step response of the reduced order system model (36), by using the integral square error (ISE) or some other integral criterion [39].

After carrying out the optimization procedure in the nominal operating point $\left(I_{\text {out }}=I_{\text {out } \max }=9 \mathrm{~A}\right)$ by using the ISE criterion, the parameters are obtained:

$$
\begin{aligned}
\omega_{0,9} & =3051.6 \mathrm{~s}^{-1}, \\
\zeta_{9} & =0.38 .
\end{aligned}
$$

The experimentally recorded step response of the feedback voltage and the step response of the reduced model (36) with parameters (37) are shown in Fig. 9. It is seen from the responses shown that the maximum error of approximation equals $5.7 \%$, which is an acceptable error value in practical applications.

After applying the same optimization procedure in the operating point determined by the lowest output current $I_{\text {out }}=$ $I_{\text {out } \min }=1 \mathrm{~A}$, the parameters are obtained:

$$
\begin{aligned}
\omega_{0,1} & =2174.3 \mathrm{~s}^{-1}, \\
\zeta_{1} & =0.462 .
\end{aligned}
$$

The experimentally recorded step response of the feedback voltage and step response of the reduced model (36) with
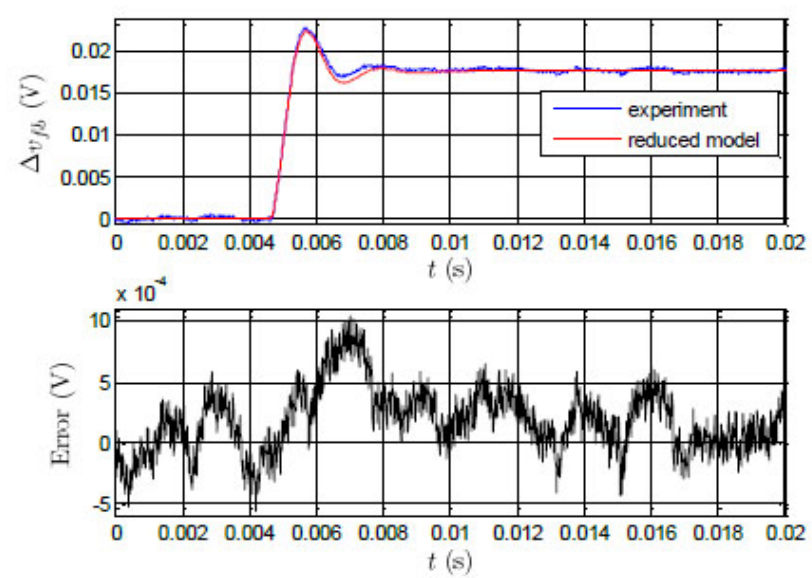

Fig. 9. The experimentally recorded step response of the feedback voltage and step response of the reduced model (36) with parameters (37), with the change of $\Delta u_{r}=0.0176 \mathrm{~S}(t-0.0046)$, and the error of approximation.
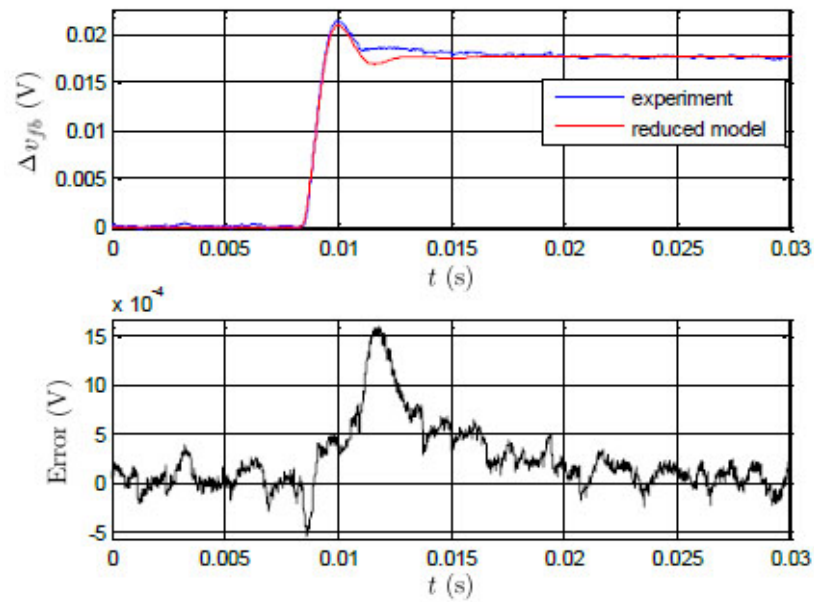

Fig. 10. The experimentally recorded step response of the feedback voltage and step response of the reduced model (36) with parameters (38), with the change of $\Delta u_{r}=0.0176 \mathrm{~S}(t-0.0084)$, and the error of approximation.

parameters (38) are shown in Fig. 10. The maximum error of approximation equals $9.1 \%$ in this case, which is also an acceptable error value in practical applications (less than $10 \%)$.

The behavior of the process in the nominal operating point represents the referent (desired) behavior of the process (adjustable system), so the reference model is designed to describe the behavior of the process precisely in the nominal operating point $\left(I_{\text {out }}=I_{\text {out } \max }=9 \mathrm{~A}\right)$. The opposite boundary operating point represents the point in which the deviation from the referent (desired) behavior is the largest ( $I_{\text {out }}=$ $I_{\text {out,min }}=1 \mathrm{~A}$ ). The converter in the nominal operating point works in a continuous conduction mode, while in the opposite boundary operating point $\left(I_{\text {out }}=I_{\text {out,min }}=1 \mathrm{~A}\right)$ it works in a discontinuous conduction mode. 


\section{Application OF THE REduced ORder Signal ADAPTATION ALGORITHM}

In this section, a procedure of model reference adaptive controller with reduced order signal adaptation algorithm design is proposed. The adaptive controller is designed for the control of processes consisting of the fuel cell BCS 64-32 (emulator in the experimental setup) and PCM boost converter, both described in detail in [24]. A part of the process is the basic PI controller, designed in Section II. The procedure of the adaptive controller design is described for the adaptive algorithm in the outer loop (Fig. 6).

After applying the model order reduction for the process with the basic controller (section III-C), its transfer function is described by (36).

The parameters of the transfer function (36) $\omega_{0}$ and $\zeta$ are changeable, depending on the operating point. For the operating point determined by $I_{\text {out }}=I_{\text {out, } \min }=1 \mathrm{~A}$, parameters $\omega_{0}$ and $\zeta$ are determined by using (38), while for the nominal operating point $I_{\text {out }}=I_{\text {out, } \max }=9 \mathrm{~A}$, parameters $\omega_{0}$ and $\zeta$ are determined by means of (37). For any other operating point in that range $I_{\text {out }} \in[1,9] \mathrm{A}$, the natural frequency of undamped oscillations is in the range $\omega_{0} \in[2174.3,3051.6] \mathrm{s}^{-1}$, and the relative damping coefficient is in the range $\zeta \in[0.462,0.38]$ (falling when $I_{\text {out }}$ rises).

The description of the process with the basic controller (36) in the state space, with the choice of state variables $x_{1}=v_{f b}$ and $x_{2}=\dot{v}_{f b}$ is given by:

$$
\left[\begin{array}{l}
\dot{x}_{1}(t) \\
\dot{x}_{2}(t)
\end{array}\right]=\left[\begin{array}{cc}
0 & 1 \\
-\omega_{0}^{2} & -2 \zeta \omega_{0}
\end{array}\right] \cdot\left[\begin{array}{l}
x_{1}(t) \\
x_{2}(t)
\end{array}\right]+\left[\begin{array}{c}
0 \\
\omega_{0}^{2}
\end{array}\right] \cdot u(t),
$$

where: $u$ - input signal of the process, consisting of the reference signal $u_{r}$ and the adaptation signal $u_{A}$ :

$$
u(t)=u_{r}(t)+u_{A}(t)
$$

The reference model is chosen to describe the behavior of the process in the nominal operating point as accurately as possible. Therefore, its transfer function is determined by (Section III-C):

$$
G_{M}(s)=\frac{\Delta v_{f b, M}(s)}{\Delta u_{r}(s)}=\frac{\omega_{M 0}^{2}}{s^{2}+2 \zeta_{M} \omega_{M 0} s+\omega_{M 0}^{2}},
$$

where: $\omega_{M 0}=\omega_{0,9}=3051.6$ and $\zeta_{M}=\zeta_{9}=0.38$.

The description of the reference model (41) in the state space, with the choice of state variables $x_{M 1}=v_{f b, M}$ and $x_{M 2}=\dot{v}_{f b, M}$, is given by:

$$
\left[\begin{array}{l}
\dot{x}_{M 1}(t) \\
\dot{x}_{M 2}(t)
\end{array}\right]=\left[\begin{array}{cc}
0 & 1 \\
-\omega_{M 0}^{2} & -2 \zeta_{M} \omega_{M 0}
\end{array}\right]\left[\begin{array}{l}
x_{M 1}(t) \\
x_{M 2}(t)
\end{array}\right]+\left[\begin{array}{c}
0 \\
\omega_{M 0}^{2}
\end{array}\right] u_{r}(t)
$$

The adaptation signal $u_{A}$ is a saturation function (with a unity gain of the linear region, $K_{v}=1$ ) of the generalized error $v$ :

$$
u_{A}(t)=\operatorname{sat}(v(t), h)
$$

where $h$ - saturation limit, and the generalized error is given by:

$$
v(t)=d_{1} \cdot\left(x_{M 1}-x_{1}\right)+d_{2} \cdot\left(x_{M 2}-x_{2}\right) .
$$

If the adaptive system is operating in the linear region of the saturation function, the adaptation signal can be written as:

$$
u_{A}(t)=d_{1} \cdot\left(x_{M 1}-x_{1}\right)+d_{2} \cdot\left(x_{M 2}-x_{2}\right),
$$

where: $d_{1}$ and $d_{2}$ - error weighting coefficients of the adaptive algorithm that need to be determined.

By inserting (45) into (40), then (40) into (39), and merging the state space descriptions (39) and (42), the complete state space form of the adaptive system is obtained:

$$
\begin{aligned}
& {\left[\begin{array}{c}
\dot{x}_{1}(t) \\
\dot{x}_{2}(t) \\
\dot{x}_{M 1}(t) \\
\dot{x}_{M 2}(t)
\end{array}\right]=\mathbf{A}_{c l} \cdot\left[\begin{array}{c}
x_{1}(t) \\
x_{2}(t) \\
x_{M 1}(t) \\
x_{M 2}(t)
\end{array}\right]+\left[\begin{array}{c}
0 \\
\omega_{0}^{2} \\
0 \\
\omega_{M 0}^{2}
\end{array}\right] \cdot u_{r}(t),} \\
& y(t)=\left[\begin{array}{llll}
1 & 0 & 0 & 0
\end{array}\right] \cdot\left[\begin{array}{c}
x_{1}(t) \\
x_{2}(t) \\
x_{M 1}(t) \\
x_{M 2}(t)
\end{array}\right]+[0] \cdot u_{r}(t),
\end{aligned}
$$

where:

$$
\mathbf{A}_{c l}=\left[\begin{array}{cccc}
0 & 1 & 0 & 0 \\
-\left(1+d_{1}\right) \omega_{0}^{2} & -\left(2 \zeta \omega_{0}+d_{2} \omega_{0}^{2}\right) & d_{1} \omega_{0}^{2} & d_{2} \omega_{0}^{2} \\
0 & 0 & 0 & 1 \\
0 & 0 & -\omega_{M 0}^{2} & -2 \zeta_{M} \omega_{M 0}
\end{array}\right] .
$$

The transfer function of the complete adaptive system (46) is given by:

$$
\begin{aligned}
& \frac{Y(s)}{U_{r}(s)}= \\
& \frac{\omega_{0}^{2}\left[s^{2}+\left(d_{2} \omega_{M 0}^{2}+2 \zeta_{M} \omega_{M 0}\right) s+\left(1+d_{1}\right) \omega_{M 0}^{2}\right]}{\left(s^{2}+2 \zeta_{M} \omega_{M 0} s+\omega_{M 0}^{2}\right)\left[s^{2}+\left(2 \zeta \omega_{0}+d_{2} \omega_{0}^{2}\right) s+\left(1+d_{1}\right) \omega_{0}^{2}\right]} .
\end{aligned}
$$

With positive process and reference model parameters $\left(\omega_{0}\right.$ $>0, \zeta>0, \omega_{M 0}>0$ and $\zeta_{M}>0$ ), the necessary stability conditions of the adaptive system are easily obtained:

$$
\begin{aligned}
& d_{1}>-1, \\
& d_{2}>-\frac{2 \zeta}{\omega_{0}} .
\end{aligned}
$$

Considering the variability of the process parameters $\omega_{0}$ and $\zeta$, the minimal value of the coefficient $d_{2}$ is determined for the worst case, which applies when $\zeta=\zeta_{\min }$ and $\omega_{0}=\omega_{0, \max }$. For the given process parameters, the conditions for the error weighting coefficients are obtained:

$$
\begin{aligned}
& d_{1}>-1, \\
& d_{2}>-2.49 \cdot 10^{-4} .
\end{aligned}
$$




\section{A. Determination of optimal values of error weighting coefficients of the adaptive algorithm}

In this paper a combined procedure based on the placement of poles and zeros and an optimization method is proposed for the determination of optimal values of error weighting coefficients of the adaptive algorithm. The procedure is described below.

It is proposed that the adaptive system is designed for the operating point which is the farthest from the nominal. In this case the boundary operating point is determined by $I_{\text {out }}=$ $I_{\text {out,min }}=1 \mathrm{~A}$, and the reduced model process parameters are $\omega_{0}=2174.3$ and $\zeta=0.462$.

The adaptive system described by the transfer function (47) has four poles and two zeros:

$$
\begin{aligned}
p_{1,2} & =\frac{-2 \zeta \omega_{0}-d_{2} \omega_{0}^{2} \pm \sqrt{\left(2 \zeta \omega_{0}+d_{2} \omega_{0}^{2}\right)^{2}-4\left(1+d_{1}\right) \omega_{0}^{2}}}{2}, \\
p_{3,4} & =\frac{-2 \zeta_{M} \omega_{M 0} \pm \sqrt{\left(2 \zeta_{M} \omega_{M 0}\right)^{2}-4 \omega_{M 0}^{2}}}{2}, \\
z_{1,2} & =\frac{-2 \zeta_{M} \omega_{M 0}-d_{2} \omega_{M 0}^{2}}{2} \pm \\
& \pm \frac{\sqrt{\left(2 \zeta_{M} \omega_{M 0}+d_{2} \omega_{M 0}^{2}\right)^{2}-4\left(1+d_{1}\right) \omega_{M 0}^{2}}}{2} .
\end{aligned}
$$

The pole-zero map of the adaptive system with $d_{1}=d_{2}=0$ (no adaptation) is shown in Fig. 11, and the responses of that (non-adaptive) system to step changes of the reference signal are shown in Fig. 12. It is evident from Fig. 11 that the poles of the reference model are canceled by the zeros of the adaptive system. Since it is desirable to achieve the best possible following of the reference model, it would be ideal to transfer the zeros to the process poles. This, of course, is not possible in such system configuration, so in this paper it is proposed that the process poles and the adaptive system zeros be transferred to the real axis of the complex s-plane in a way that the pole on the real axis closer to the origin of the coordinate system cancels the zero closer to the origin, and the remaining pole and zero are placed as far away from the origin as possible, so that they would not affect the dynamics of the adaptive system.

According to the pole-zero map $p_{1,2}$ and $z_{1,2}$ in (50), that request is achieved with:

$$
\begin{aligned}
& \left(2 \zeta \omega_{0}+d_{2} \omega_{0}^{2}\right)^{2}-4\left(1+d_{1}\right) \omega_{0}^{2} \gg 0, \\
& \left(2 \zeta_{M} \omega_{M 0}+d_{2} \omega_{M 0}^{2}\right)^{2}-4\left(1+d_{1}\right) \omega_{M 0}^{2} \gg 0,
\end{aligned}
$$

from which the following conditions are obtained:

$$
\begin{aligned}
& d_{1} \ll \frac{1}{4} \omega_{0}^{2} d_{2}^{2}+\zeta \omega_{0} d_{2}+\zeta^{2}-1, \\
& d_{1} \ll \frac{1}{4} \omega_{M 0}^{2} d_{2}^{2}+\zeta_{M} \omega_{M 0} d_{2}+\zeta_{M}^{2}-1 .
\end{aligned}
$$

The boundary cases for conditions (52) are given by:

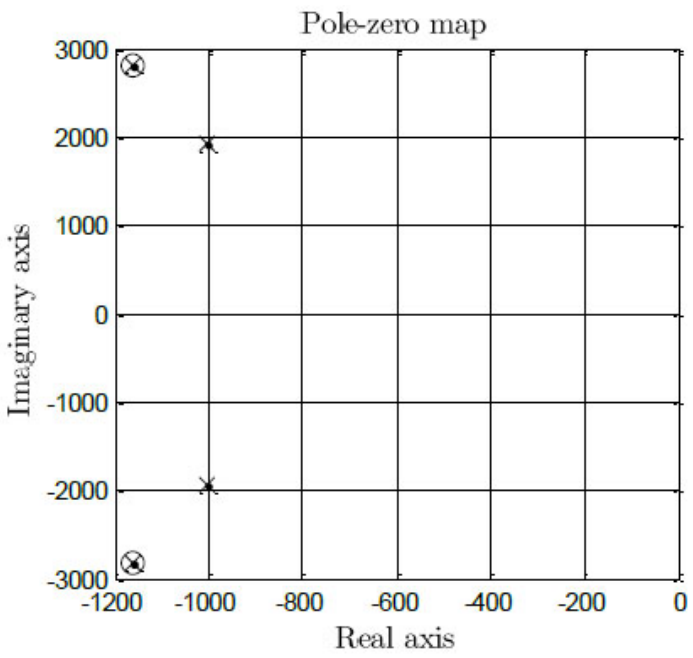

Fig. 11. The pole-zero map of the adaptive system for coefficients values $d_{1}=d_{2}=0$ (no adaptation).

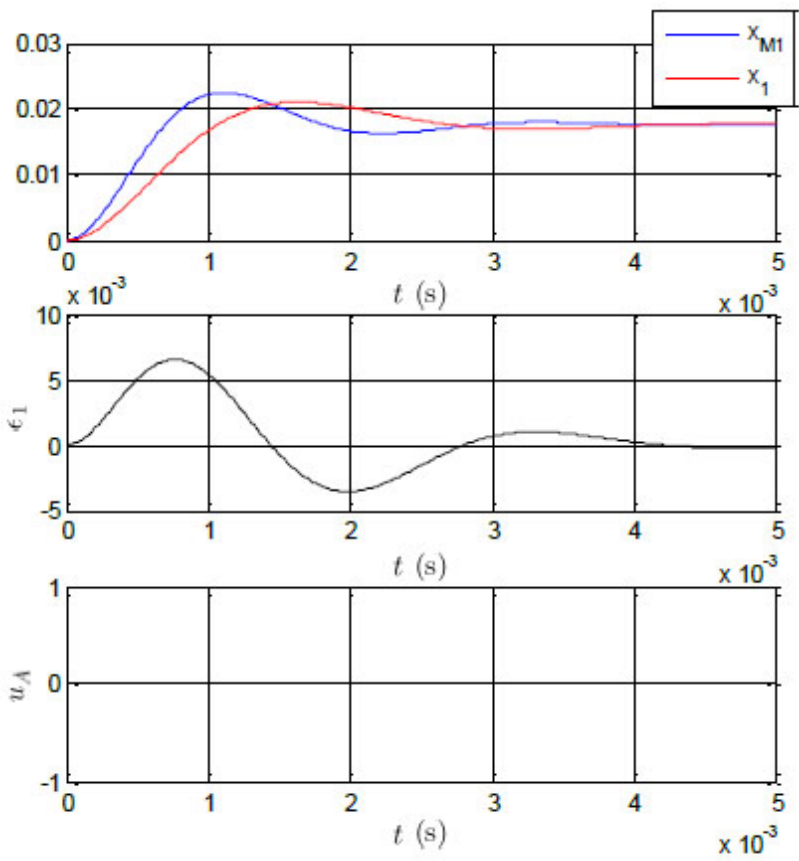

Fig. 12. Responses of the system without adaptation $\left(d_{1}=d_{2}=\right.$ 0) $x_{M 1}$ and $x_{1}$, errors $e_{1}=x_{M 1}-x_{1}$ and adaptation signal $u_{A}$, on the step change of the reference signal $u_{r}(t)=0.0176 \mathrm{~S}(t)$.

$$
\begin{gathered}
d_{1}=\frac{1}{4} \omega_{0}^{2} d_{2}^{2}+\zeta \omega_{0} d_{2}+\zeta^{2}-1, \\
d_{1}=\frac{1}{4} \omega_{M 0}^{2} d_{2}^{2}+\zeta_{M} \omega_{M 0} d_{2}+\zeta_{M}^{2}-1 .
\end{gathered}
$$

The graphical dependence of the relation of coefficients $d_{1}$ and $d_{2}$, according to (53) and (54), is shown in Fig. 13. It is evident from Fig. 13 that the request (52) is fulfilled for large values of coefficient $d_{2}$ and at least 10 times smaller value of the coefficient $d_{1}$ than the appropriate point on the lower curve in Fig. 13. 


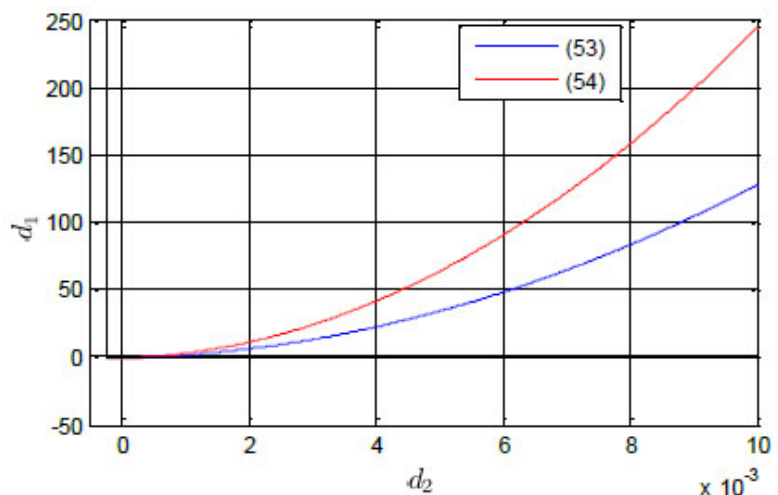

Fig. 13. The graphical dependence of relation of coefficients $d_{1}$ and $d_{2}$, according to (53) and (54).

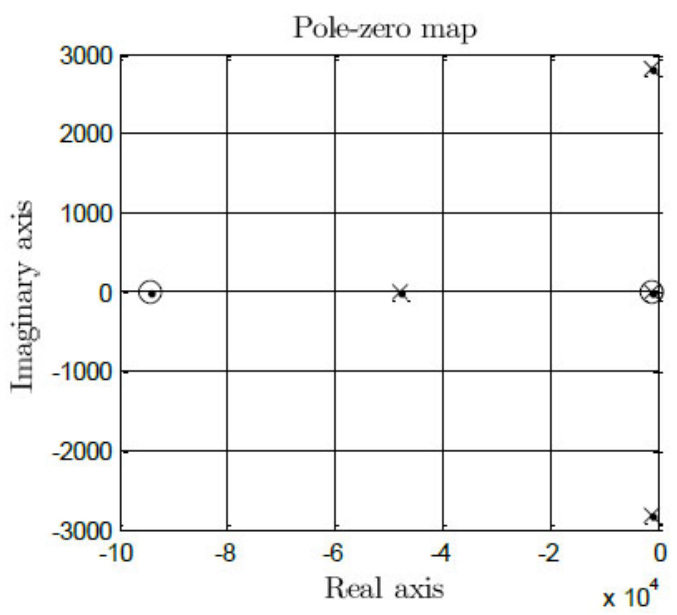

Fig. 14. The pole-zero map of the continuous adaptive system with error weighting coefficients $d_{1}=12.7$ and $d_{2}=0.01$.
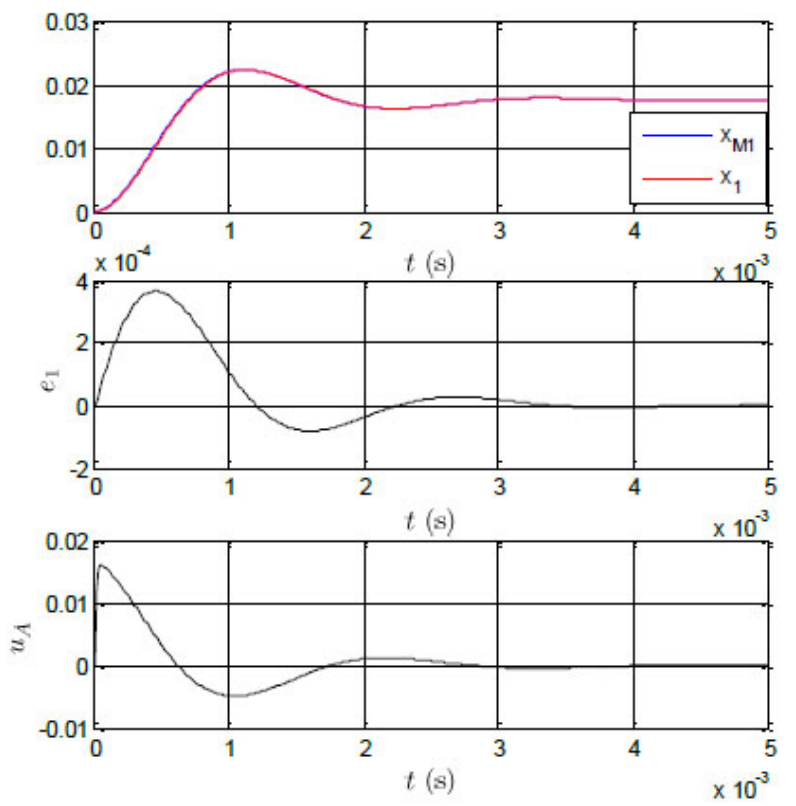

Fig. 15. Responses of the adaptive system $x_{M 1}$ and $x_{1}$, error $e_{1}=x_{M 1}-x_{1}$ and adaptation signal $u_{A}$, on the step change of the reference signal $u_{r}(t)=0.0176 \mathrm{~S}(t)$, with $d_{1}=12.7$ and $d_{2}=0.01$.
In practical applications, excessive value of coefficient $d_{2}$ causes significant amplification of the noise, because the coefficient multiplies the derivative of the error.

For example, if the coefficient $d_{2}$ is chosen to be $d_{2}=0.01$, then according to curves in Fig. 13, the coefficient $d_{1}$ has to be at least 10 times smaller than 127 . If the exact 10 times smaller value is chosen $d_{1}=12.7$, the pole-zero map of the adaptive system shown in Fig. 14 is obtained, and it shows that the request (52) is achieved, i.e. the dynamics of the adaptive system is determined by the dominant pair of the reference model poles. This is also confirmed by responses of the adaptive system to step changes of the reference signal shown in Fig. 15. As may be seen, the maximum error $e_{1, \max }$ is reduced from $e_{1, \max }=35 \%$ to approximately $e_{1, \max }=2 \%$ when using an adaptive controller.

By additional reduction of the $d_{2}$, e.g. to the value $d_{2}=$ 0.001 , the first coefficient is obtained in a similar way, and it has the value $d_{1}=0.14$. In this way the sensitivity of the adaptive algorithm to the noise is reduced. Furthermore, from responses shown in Fig. 16, it is evident that the quality of adaptation is worse than in the previous case. The maximum error is reduced from $e_{1, \max }=35 \%$ to approximately $e_{1, \max }=17 \%$.

It is obvious from all this that practical applications require a compromise between good following of the reference signal and sensitivity to noise. The value of the coefficient $d_{2}$ is therefore optimized, and the value of the coefficient $d_{1}$ is set at a value at least 10 times smaller than the appropriate value determined by (52).

In the configuration of the adaptive system shown in Fig. 6, it is proposed that the saturation limit $h$ in (25) equals 10 do $20 \%$ of the value of maximum reference signal range. In this case it is a value of $h=1$. A good design of the adaptive controller with the saturation function (25) should always ensure operation in the linear region of the saturation function.

The gain coefficient of the linear region of the saturation function $K_{v}$ is proposed to be set to unity value $K_{v}=1$, and in practical applications it can be used for reducing the oscillations that may occur in the adaptation signal.

\section{B. Simulation results}

The block schematics of the adaptive system with nonlinear models of the fuel cell BCS 64-32 and PCM boost converter, with a fast output sampling (FOS) estimator of state variables [21, 40], for the simulation in MATLAB-SimUlinK is shown in Fig. 17.

System responses without adaptation are shown in Fig. 18. The maximum error due to the change of the reference signal equals $e_{1, \max }=26.2 \%$, and the maximum feedback voltage drop due to the change of the disturbance signal 

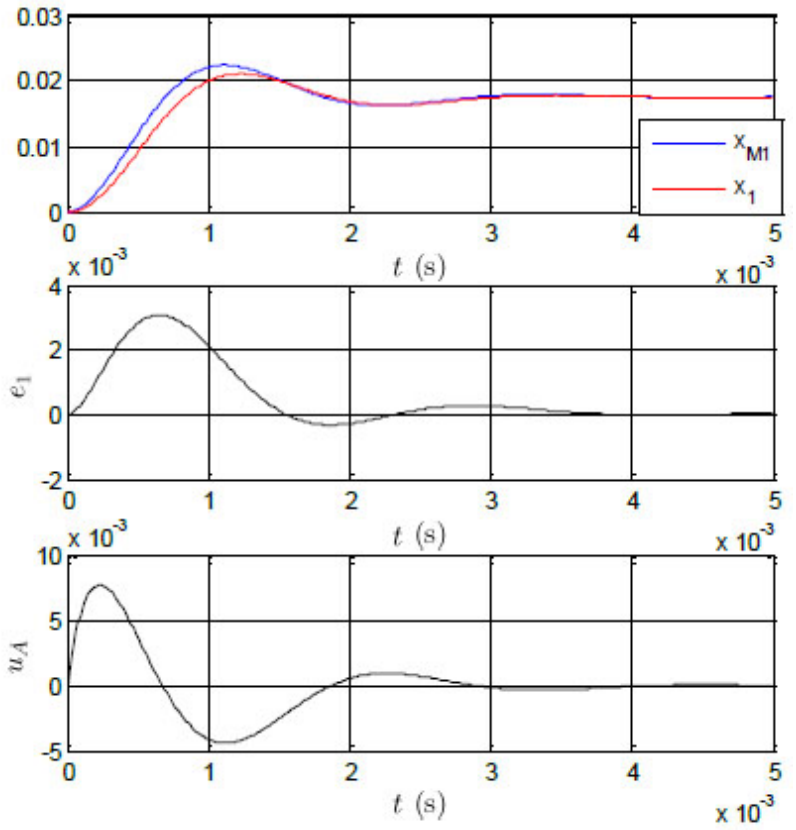

Fig. 16. Responses of the adaptive system $x_{M 1}$ and $x_{1}$, error $e_{1}=x_{M 1}-x_{1}$ and adaptation signal $u_{A}$, on the step change of the reference signal $u_{r}(t)=0.0176 \mathrm{~S}(t)$, with $d_{1}=0.14$ and $d_{2}=0.001$.

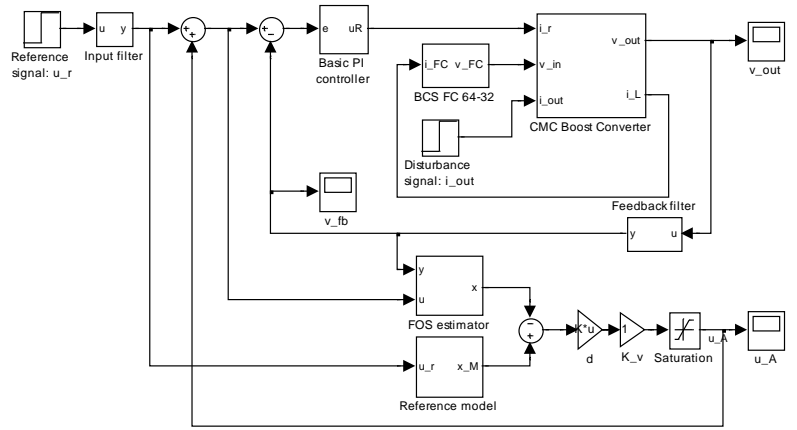

Fig. 17. The block schematics of the adaptive system with nonlinear models of the fuel cell BCS 64-32 and PCM boost converter, with FOS estimator of state variables, for the simulation in MATLAB-SIMULINK.

equals $\Delta v_{f b, \max }=0.153 \mathrm{~V}$.

The same responses of the adaptive system with coefficients $d_{1}=0.14$ and $d_{2}=0.001$ are shown in Fig. 19 . It is seen that the maximum error due to the change of reference signal is reduced to $e_{1, \max }=11.7 \%$ and the maximum feedback voltage drop due to the change of the disturbance signal is reduced to $\Delta v_{f b \text {, } \max }=0.073 \mathrm{~V}$, which is more than two times!

As already mentioned, the increase in the value of the coefficient $d_{2}$ should result in even better adaptation. More
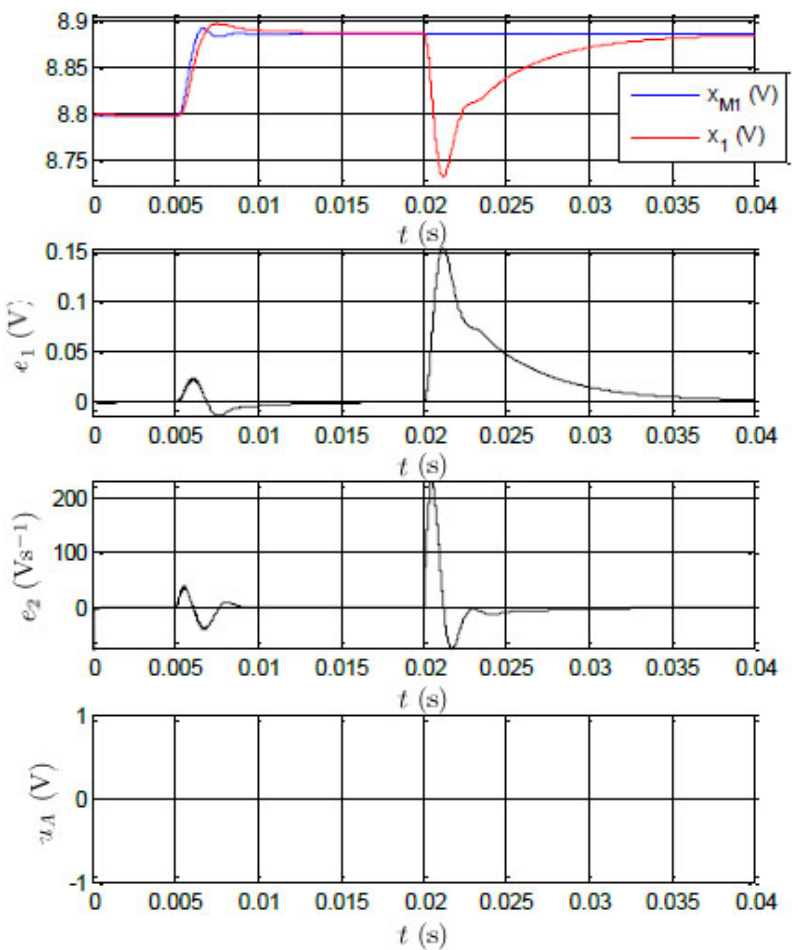

Fig. 18. System responses without adaptation ( $d_{1}=0$ and $\left.d_{2}=0\right)$ $x_{M 1}$ and $x_{1}$, errors $e_{1}=x_{M 1}-x_{1}$ and $e_{2}=x_{M 2}-x_{2}$ and adaptation signal $u_{A}$, on the step change of reference signal $u_{r}(t)=8.799+0.088 \mathrm{~S}(t) \quad \mathrm{V} \quad$ and disturbance signal $i_{\text {out }}(t)=1+8 \mathrm{~S}(t-0.02) \mathrm{A}$.

precisely, by doubling $d_{2}$ to a value of $d_{2}=0.002$ and setting the value of coefficient $d_{1}$ at a value 10 times smaller than the minimal one determined by (52), which is $d_{1}=0.59$, the responses shown in Fig. 20 are obtained. An additional reduction of errors is achieved to values $e_{1, \max }=7.9 \%$ and $\Delta v_{f b, \max }=0.044 \mathrm{~V}$, which is a reduction of almost 3.5 times. However, the beginning of oscillations in the adaptation signal is also seen, which is a potential problem for application in real systems, due to the presence of noise.

\section{Experimental results with the FOS estimator}

In the LabviEw software environment the adaptive controller with reduced order reference model and signal adaptation algorithm, and with the FOS estimator of the state variables [21, 40], is implemented for application in a National Instruments CompactRIO 9024 FPGA hardware system. The adaptive algorithm is realized with the sample time $\tau=30 \mu \mathrm{s}$.

Experimental responses of the system without adaptation are shown in Figs. 21 and 22. The maximum relevant error due to the change of reference signal equals $e_{1, \max }=16 \%$ (the error is here defined as $e_{1}=x_{M 1}-x_{1}$, where $x_{1}$ is the 

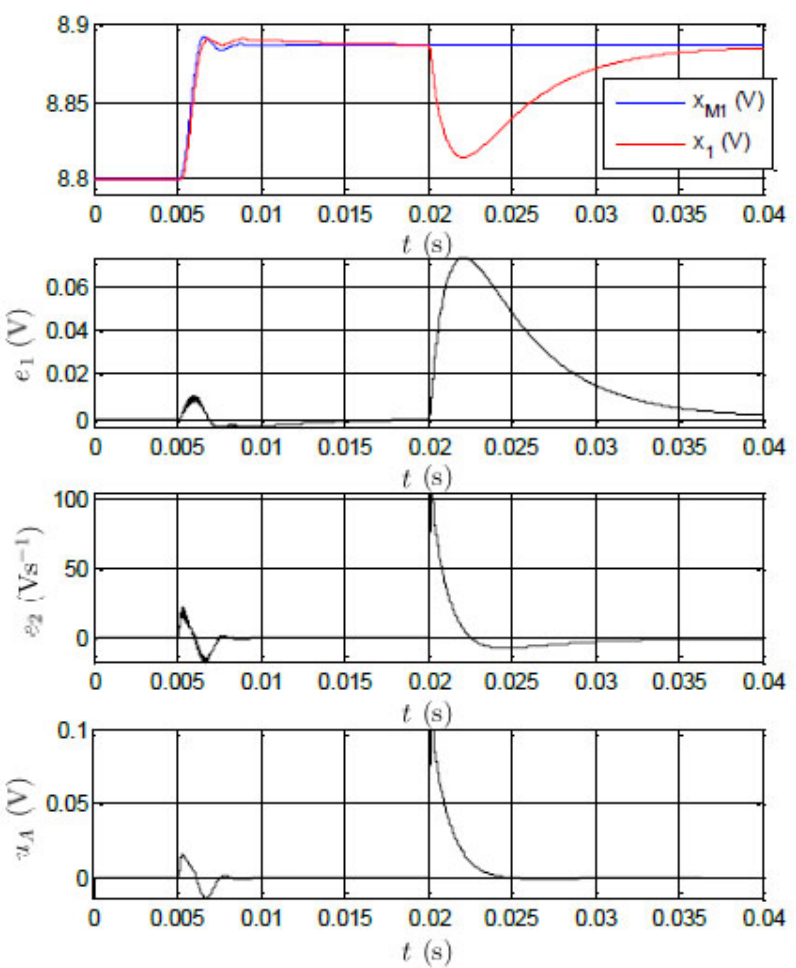

Fig. 19. Responses of the adaptive system $x_{M 1}$ and $x_{1}$, errors $e_{1}=x_{M 1}-x_{1}$ and $e_{2}=x_{M 2}-x_{2}$ and adaptation signal $u_{A}$, on the step change of reference signal $u_{r}(t)=8.799+0.088 \mathrm{~S}(t) \quad \mathrm{V} \quad$ and disturbance signal $i_{\text {out }}(t)=1+8 S(t-0.02)$ A, with $d_{1}=0.14$ and $d_{2}=0.001$.

estimated state variable). The error is larger when the basic controller is in the saturation region (due to the limited output $0-25 \mathrm{~mA}$ ), but that case is not considered because the control system is then practically in the open loop. The maximum feedback voltage drop due to the change of the disturbance signal equals $\Delta v_{f b, \max }=0.116 \mathrm{~V}$.

The same responses with the adaptation coefficients $d_{1}=0.14$ and $d_{2}=0.001$ are shown in Figs. 23 and 24 .

It is evident from these responses that the maximum relevant error due to the change of the reference signal is reduced almost 3 times to a value of $e_{1, \max }=5.7 \%$, and the maximum feedback voltage drop due to the change of the disturbance signal is reduced more than 2 times to a value of $\Delta v_{f b, \max }=0.055 \mathrm{~V}$.

Even though the analysis of the obtained results shows that the adaptation results are very good, a significant amplification of the noise in the control and adaptation signal is seen, which is not desirable. The reason for this is in asynchronism of clocks of the modulation signal and the fast output samples of the FOS estimator.

Therefore, for this application a modification is proposed to minimize the influence of the noise in the experimental setup on the quality of the adaptive control system.
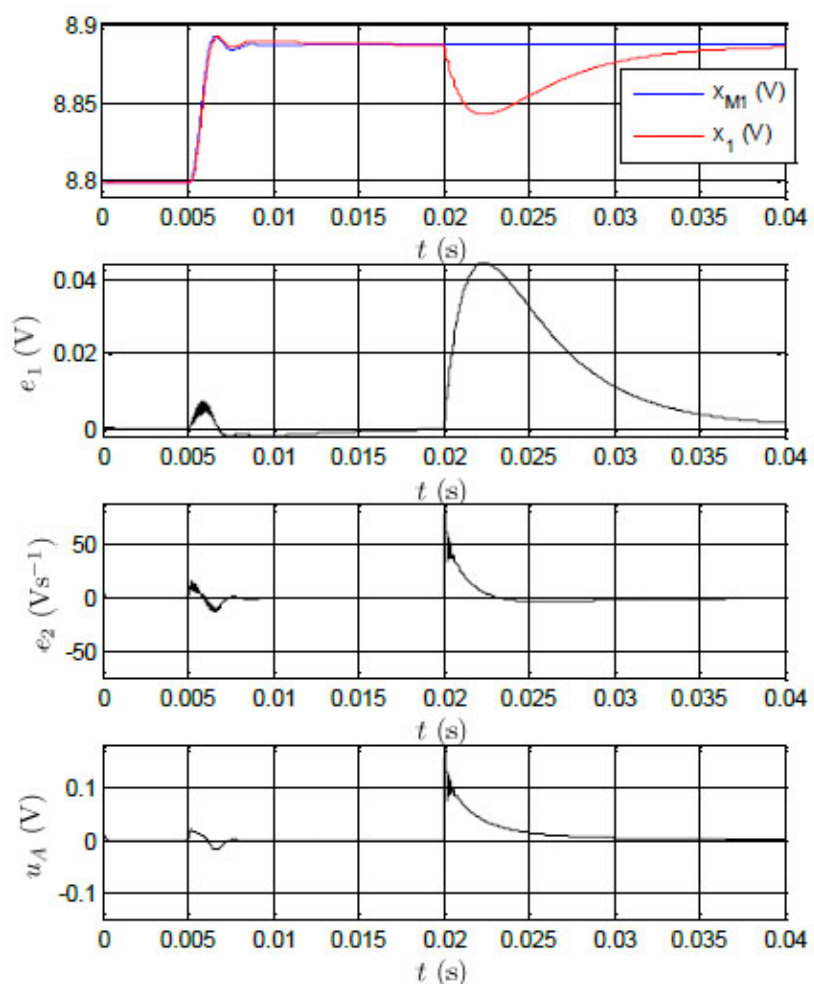

Fig. 20. Responses of the adaptive system $x_{M 1}$ and $x_{1}$, errors $e_{1}=x_{M 1}-x_{1}$ and $e_{2}=x_{M 2}-x_{2}$ and adaptation signal $u_{A}$, on the step change of reference signal $u_{r}(t)=8.799+0.088 \mathrm{~S}(t) \quad \mathrm{V} \quad$ and disturbance signal $i_{\text {out }}(t)=1+8 \mathrm{~S}(t-0.02)$ A, with $d_{1}=0.59$ and $d_{2}=0.002$.

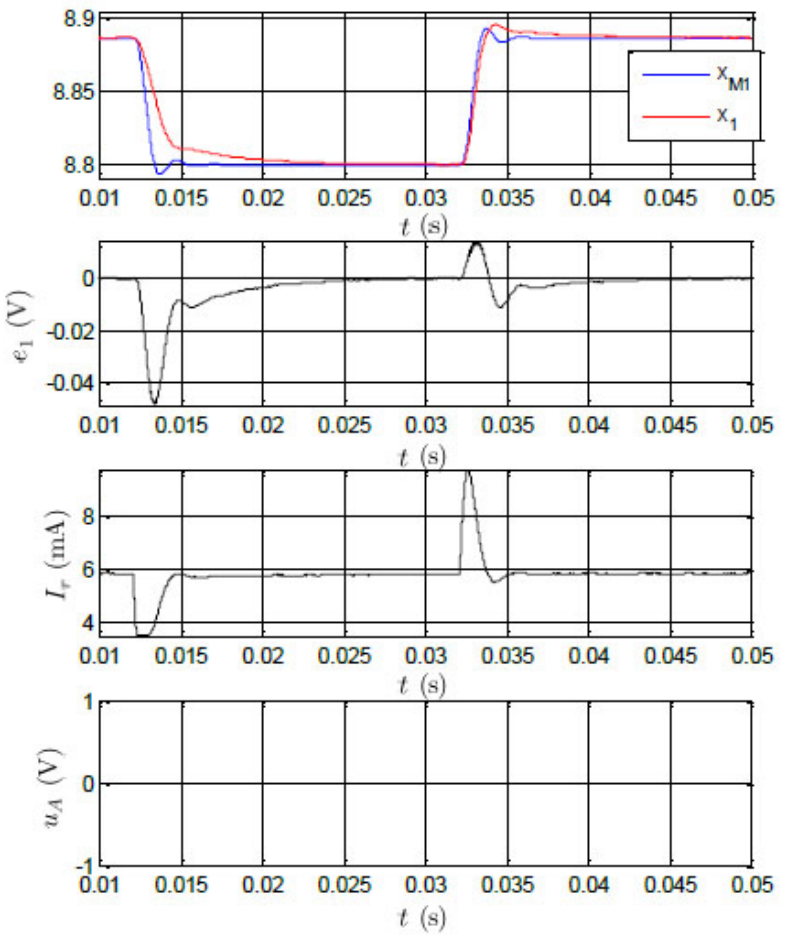

Fig. 21. Responses of the system without adaptation $\left(d_{1}=0\right.$ and $\left.d_{2}=0\right) x_{M 1}$ and $x_{1}$, error $e_{1}=x_{M 1}-x_{1}$, control signal $I_{r}$ and adaptation signal $u_{A}$, on the step changes of the reference signal $\Delta u_{r}= \pm 0.088 \mathrm{~V}$, for the operating point $I_{\text {out }}=I_{\min }=1 \mathrm{~A}$. 

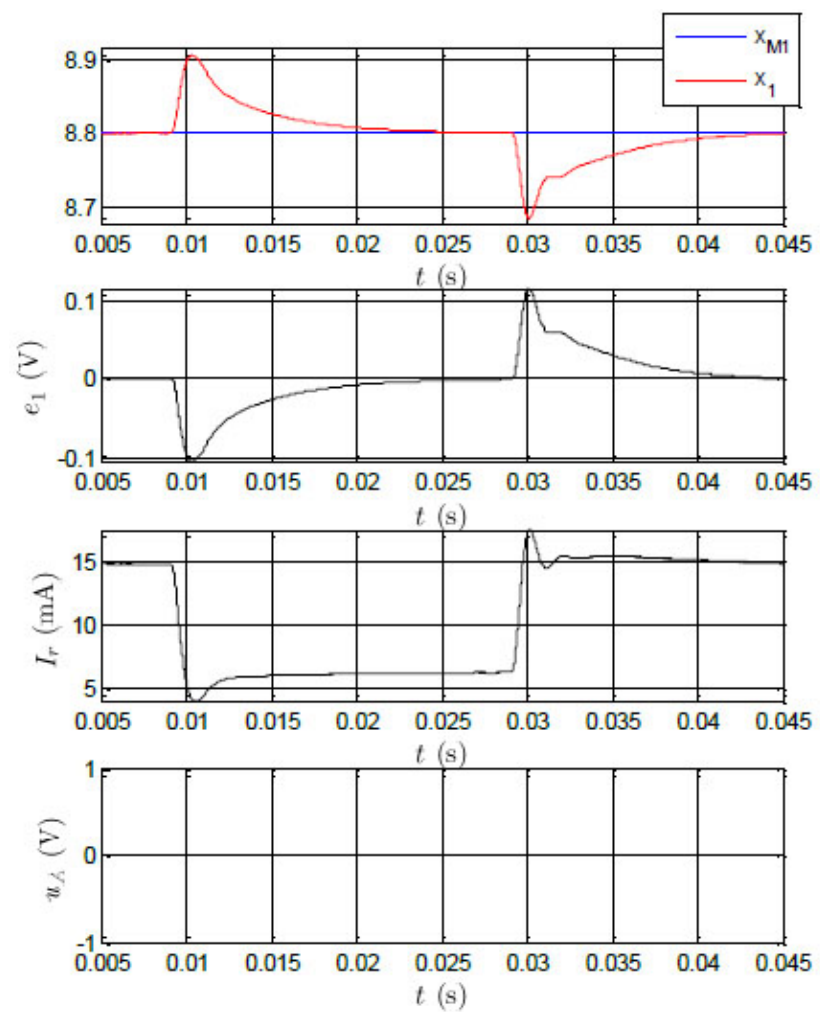

Fig. 22. Responses of the system without adaptation $\left(d_{1}=0\right.$ and $\left.d_{2}=0\right) x_{M 1}$ and $x_{1}$, error $e_{1}=x_{M 1}-x_{1}$, control signal $I_{r}$ and adaptation signal $u_{A}$, on the step changes of the disturbance signal $\Delta i_{\text {out }}= \pm 8 \mathrm{~A}$.
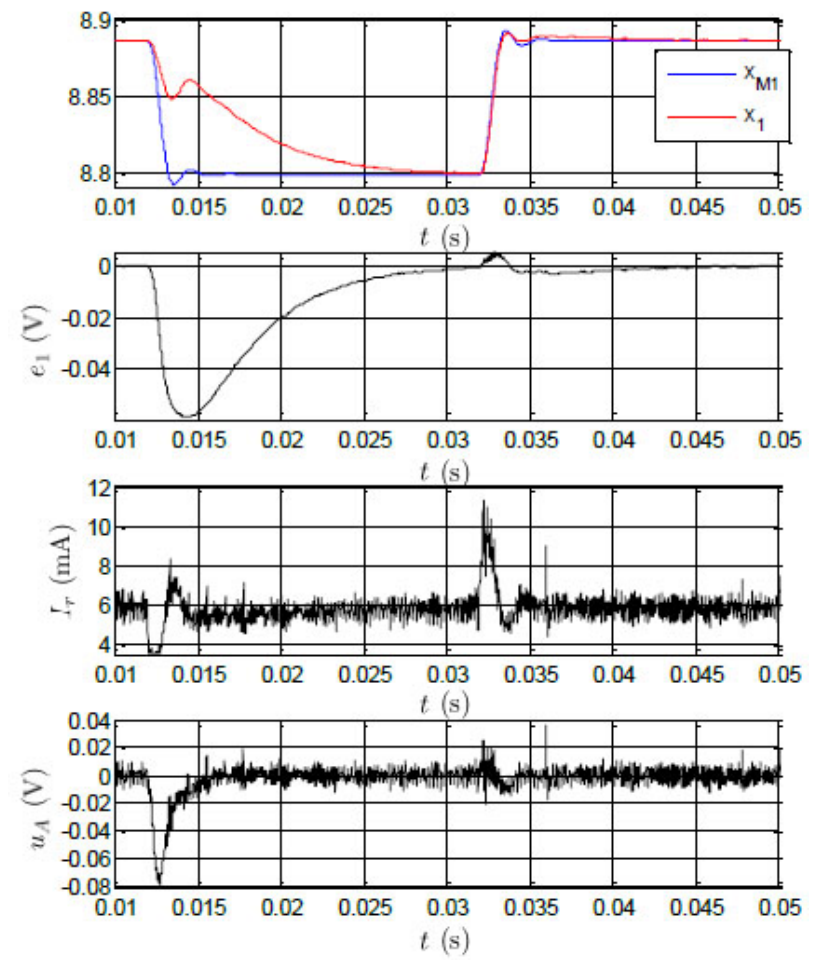

Fig. 23. Responses of the adaptive system $x_{M 1}$ and $x_{1}$, error $e_{1}=x_{M 1}-x_{1}$, control signal $I_{r}$ and adaptation signal $u_{A}$, on the step changes of the reference signal $\Delta u_{r}= \pm 0.088 \mathrm{~V}$, with $d_{1}=0.14$ and $d_{2}=0.001$ for the operating point $I_{\text {out }}=I_{\min }=1 \mathrm{~A}$.
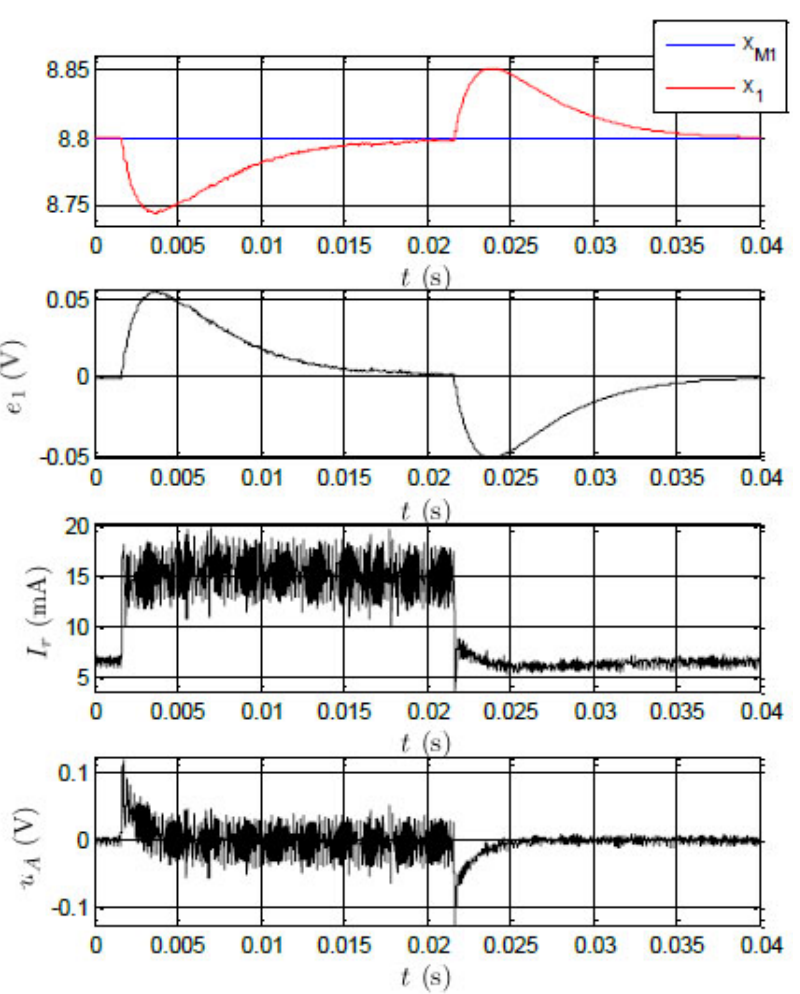

Fig. 24. Responses of the adaptive system $x_{M 1}$ and $x_{1}$, error $e_{1}=x_{M 1}-x_{1}$, control signal $I_{r}$ and adaptation signal $u_{A}$, on the step changes of the disturbance signal $\Delta i_{\text {out }}= \pm 8 \mathrm{~A}$, with $d_{1}=0.14$ and $d_{2}=0.001$.

D. Experimental results with a derivative of the feedback voltage

The responses to the change of reference signal, shown in the previous subsection IV-C, were strongly influenced by process and measurement noise in the adaptive system. This influence caused that step changes of referent output voltage had to be set to a value of $\Delta u_{r}= \pm 0.088 \mathrm{~V}\left(\Delta v_{\text {out }}= \pm 0.5 \mathrm{~V}\right)$. Thereby, the basic PI controller output, in the operating point determined by $I_{\text {out }}=1 \mathrm{~A}$, is in the saturation region, when the referent voltage steps by $-0.5 \mathrm{~V}$. Using the system output signal and its derivative as state variables, the estimator can be avoided. The use of the real feedback voltage derivative allows the reduction of the noise influence to the responses of the adaptive system. Therefore, recorded experimental transients with a much smaller change of the reference signal $\Delta u_{r}= \pm 0.0176 \mathrm{~V}\left(\Delta v_{\text {out }}= \pm 0.1 \mathrm{~V}\right)$ could be obtained. In that way the basic PI controller does not enter the saturation region.

The transfer function of the real derivative block is given by:

$$
G_{r d}(s)=\frac{\dot{V}_{f b}(s)}{V_{f b}(s)}=\frac{s}{1+T_{v} s},
$$

where: $T_{v}$ - parasite time constant. 

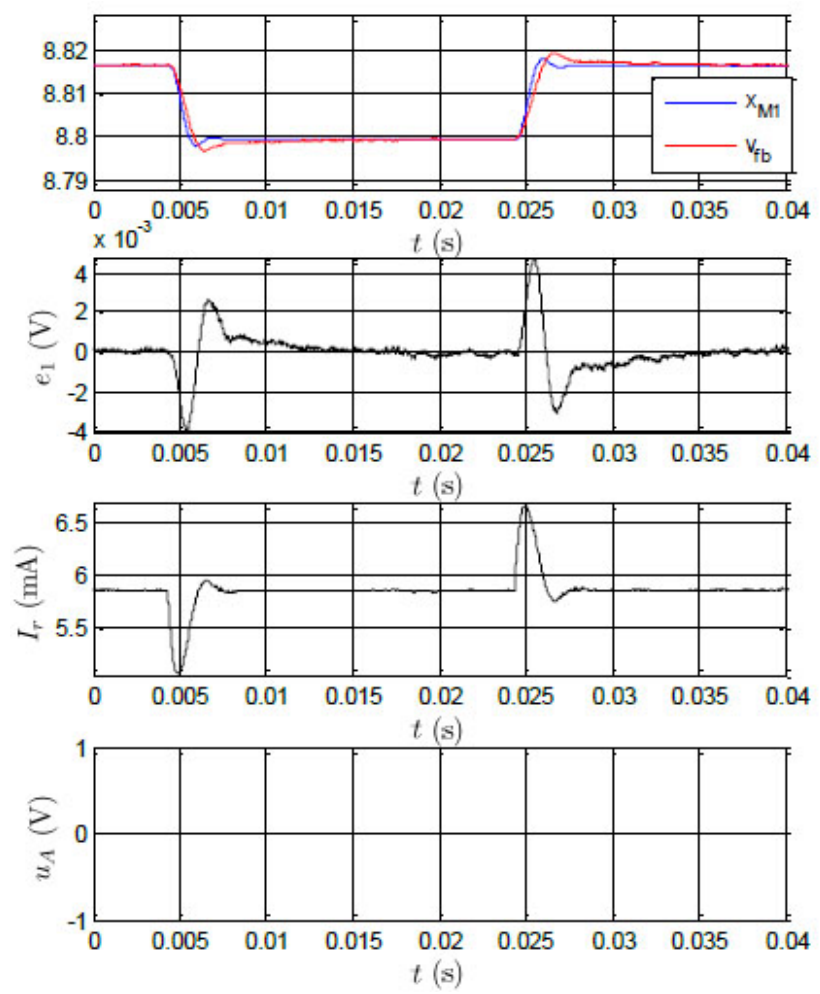

Fig. 25. Responses of the system without adaptation $\left(d_{1}=0\right.$ and $\left.d_{2}=0\right) x_{M 1}$ and $x_{1}$, error $e_{1}=x_{M 1}-x_{1}$, control signal $I_{r}$ and adaptation signal $u_{A}$, on the step changes of the reference signal $\Delta u_{r}= \pm 0.0176 \mathrm{~V}$, for the operating point $I_{\text {out }}=I_{\min }=1 \mathrm{~A}$.

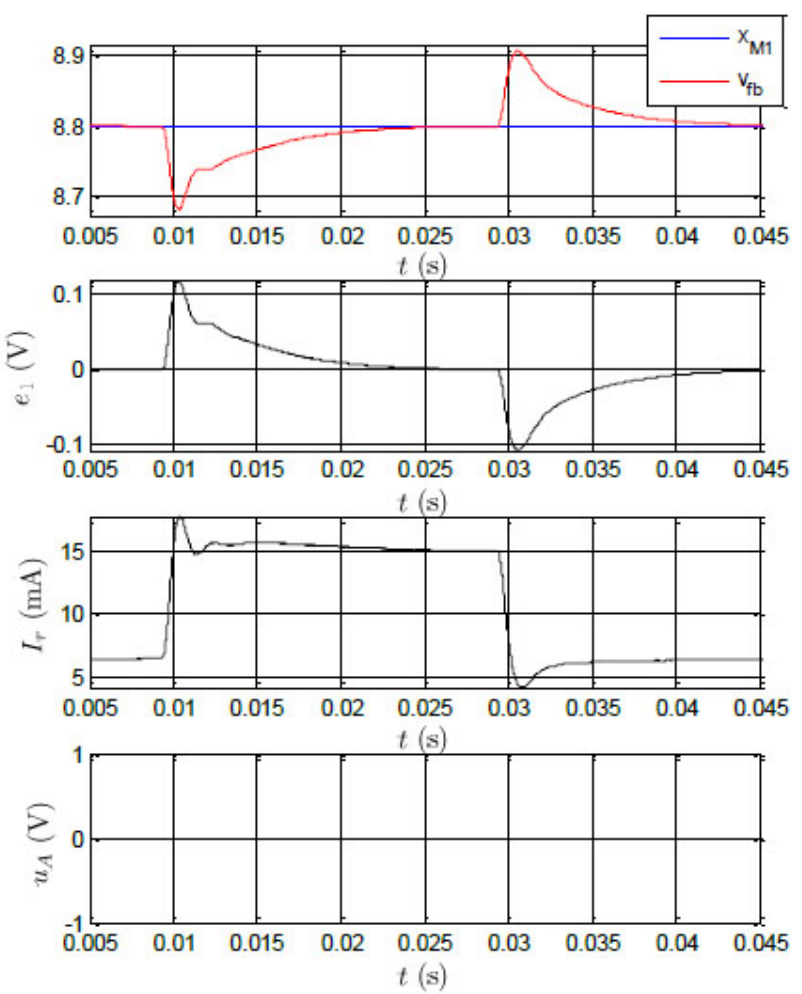

Fig. 26. Responses of the system without adaptation $\left(d_{1}=0\right.$ and $\left.d_{2}=0\right) x_{M 1}$ and $x_{1}$, error $e_{1}=x_{M 1}-x_{1}$, control signal $I_{r}$ and adaptation signal $u_{A}$, on the step changes of the disturbance signal $\Delta i_{\text {out }}= \pm 8 \mathrm{~A}$.
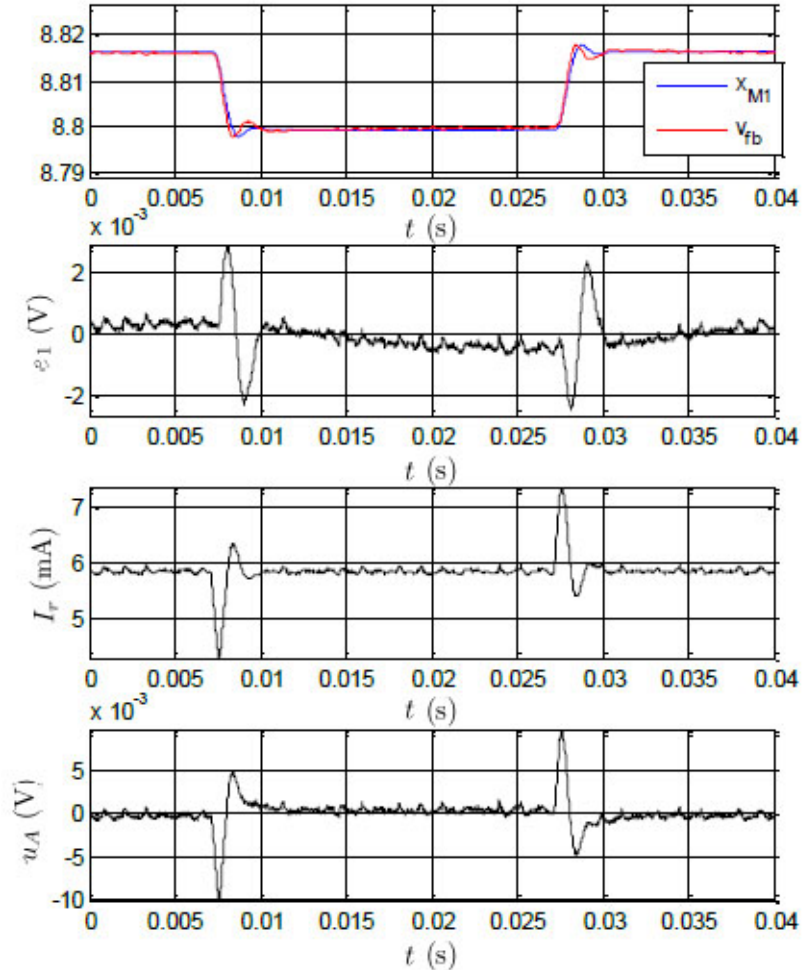

Fig. 27. Responses of the adaptive system $x_{M 1}$ and $x_{1}$, error $e_{1}=x_{M 1}-x_{1}$, control signal $I_{r}$ and adaptation signal $u_{A}$, on the step changes of the reference signal $\Delta u_{r}= \pm 0.0176 \mathrm{~V}$, with $d_{1}=0.14$ and $d_{2}=0.001$ for the operating point $I_{\text {out }}=I_{\min }=1 \mathrm{~A}$.
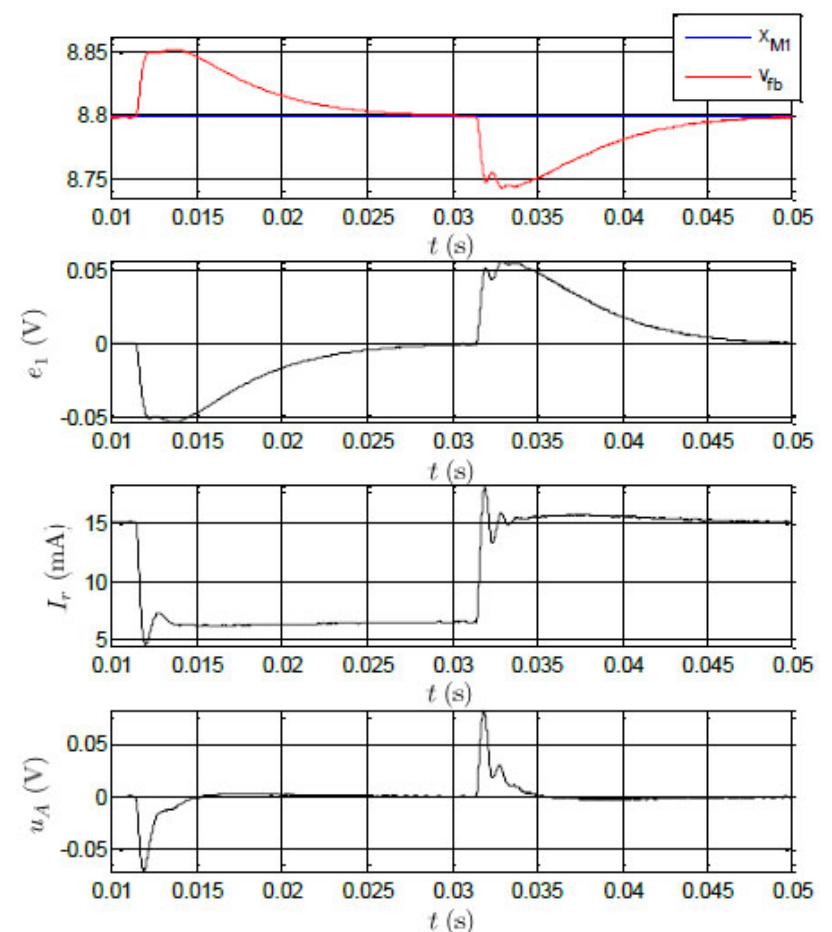

Fig. 28. Responses of the adaptive system $x_{M 1}$ and $x_{1}$, error $e_{1}=x_{M 1}-x_{1}$, control signal $I_{r}$ and adaptation signal $u_{A}$, on the step changes of the disturbance signal $\Delta i_{\text {out }}= \pm 8 \mathrm{~A}$, with $d_{1}=0.14$ and $d_{2}=0.001$. 
The value of the parasite time constant is determined by experimenting in a few iterations. It must be large enough to reduce the noise as much as possible, and at the same time retain the good derivative of the feedback voltage. For the adaptive system with the fuel cell emulator and PCM boost converter the optimal value is $T_{v}=400 \mu$. With the sample time of the adaptive algorithm being $T_{s}=15 \mu$, the transfer function in the discrete domain is obtained by zero order hold $(\mathrm{ZOH})$ discretization, which is suitable for implementation in a National Instruments CompactRIO 9024 FPGA hardware system:

$$
G_{r d, d}(z)=\frac{\dot{V}_{f b}(z)}{V_{f b}(z)}=2500 \cdot \frac{z-1}{z-0.963194418} .
$$

Experimental results of the system responses without adaptation are shown in Figs. 25 and 26. The maximum error due to the change of referent signal equals $e_{1, \max }=26.7 \%$, and the maximum feedback voltage drop due to the change of the disturbance signal equals $\Delta v_{f b, \max }=0.117 \mathrm{~V}$.

The responses of the adaptive system in the same conditions, with coefficients $d_{1}=0.14$ and $d_{2}=0.001$ are shown in Figs. 27 and 28. It is evident from the responses that maximum error due to the change of the reference signal is reduced to $e_{1, \max }=16.3 \%$, and the maximum feedback voltage drop due to the change of the disturbance signal is reduced more than 2 times to a value of $\Delta v_{f b, \max }=0.056 \mathrm{~V}$. The good properties of the adaptive system are retained, and at the same time the noise is significantly reduced.

\section{CONCLUSION}

In this paper the adaptive control of the peak current mode controlled (PCM) boost converter supplied by the PEM fuel cell is described.

The main contributions of the paper are summarized below:

i. a reduced order adaptive algorithm with the reference model and signal adaptation algorithm is derived,

ii. the procedure for determining optimal error weighting coefficients of the adaptive algorithm, based on the combination of the pole-zero placement and an optimization method is described.

The comparative advantage of the described adaptive algorithm of reduced order with respect to other advanced control methods is in simple and straightforward determination of optimal coefficients of the adaptive algorithm. Optimal values of error weighting coefficients can be determined in just a few iterations, without the use of the computer. Therefore, the procedure is very suitable for implementation in real systems, not just the boost converter supplied by the fuel cell.

Simulation and experimental results show that the dynamic behavior of a highly nonlinear control system with a fuel cell and a PCM boost converter can fairly accurately be described in either the continuous or the discontinuous conduction mode by the dynamic behavior of the reference model, i.e., linear system with constant parameters.

\section{ACKNOWLEDGMENTS}

This research has been funded by the Ministry of Science, Education and Sports of the Republic of Croatia.

The authors would like to thank the Mareton d.o.o., Croatian Power Electronics company for the design and construction of the special version of the boost converter, which enabled the experimental verification of the adaptive control algorithm.

\section{REFERENCES}

[1] J. M. Andújar, F. Segura, and M. J. Vasallo, “A suitable model plant for control of the set fuel cell-DC/DC converter," Renewable Energy, Vol. 33, No. 4, pp. 813-826, Apr. 2008.

[2] W. Choi, J. W. Howze, and P. Enjeti, "Fuel-cell powered uninterruptible power supply systems: Design considerations," Journal of Power Sources, Vol. 157, No. 1, pp. 311-317, Jun. 2006.

[3] R. Dufo-López, J. L.Bernal-Agustín, and J. Contreras, "Optimization of control strategies for stand-alone renewable energy systems with hydrogen storage," Renewable Energy, Vol. 32, No. 7, pp. 1102-1126, Jun. 2007.

[4] K. Jin, X. Ruan, M. Yang, and M. Xu, “A hybrid fuel cell power system,” IEEE Trans. Ind. Electron., Vol. 56, No. 4, pp. 1212-1222, Apr. 2009.

[5] A. Kirubakaran, S. Jain, and R. K. Nema, "A review on fuel cell technologies and power electronic interface,” Renewable and Sustainable Energy Reviews, Vol. 13, No. 9, pp. 2430-2440, Dec. 2009.

[6] V. Paladini, T. Donateo, A. de Risi, and D. Laforgia, "Super-capacitors fuel-cell hybrid electric vehicle optimization and control strategy development," Energy Conversion and Management, Vol. 48, No. 11, pp. 3001-3008, Nov. 2007.

[7] F. Segura, E. Durán, and J. M. Andújar, "Design, building and testing of a stand alone fuel cell hybrid system," Journal of Power Sources, Vol. 193, No. 1, pp. 276-284, Aug. 2009.

[8] P. Thounthong and B. Davat, "Study of a multiphase interleaved step-up converter for fuel cell high power applications,” Energy Conversion and Management, Vol. 51, No. 4, pp. 826-832, Apr. 2010.

[9] P. Thounthong and S. Raël, "The benefits of hybridization: An investigation of fuel cell/battery and fuel cell/supercapacitor hybrid sources for vehicle 
applications,” IEEE Industrial Electronics Magazine Vol. 3, No. 3, pp. 25-37, Sep. 2009.

[10] P. Thounthong, P. Sethakul, S. Raël, and B. Davat, "Control of fuel cell/battery/supercapacitor hybrid source for vehicle applications," in Proc. ICIT, pp. 1-6, Feb. 2009.

[11] M. Uzunoglu, O. C. Onar, and M. S. Alam, "Modeling, control and simulation of a PV/FC/UC based hybrid power generation system for stand-alone applications," Renewable Energy, Vol. 34, No. 3, pp. 509-520, Mar. 2009.

[12] Y. -. Yang, Z. -. Liu, and F. -. Wang, "An application of indirect model reference adaptive control to a low-power proton exchange membrane fuel cell," Journal of Power Sources, Vol. 179, No. 2, pp. 618-630, May 2008.

[13] T. Bjažić, Ž. Ban, and I. Volarić, "Control of a fuel cell stack loaded with DC/DC boost converter," in Proc. ISIE, pp. 1489-1494, Jun./Jul. 2008.

[14] G. Ji, F. Chen, T. Ma, C. Zhang, Y. Zhou, and S. Zhou, "Development of a model-based controller prototype for a PEM fuel cell engine with ASCET-MD," in Proc. ICCA, pp. 2100-2105, Dec. 2009.

[15] J. Jia, S. Yang, Y.Wang, and Y. T. Cham, "Matlab/Simulink based-study on PEM fuel cell and nonlinear control,” in Proc. ICCA, pp. 1657-1662, Dec. 2009.

[16] F. Khorrami, S. Puranik, A. Keyhani, P. Krishnamurthy, and Y. She, "PEM fuel cell distributed generation system: Modeling and robust nonlinear control," in Proc. CDC/CCC, pp. 7860-7865, Dec. 2009.

[17] E.-S. Kim, and C.-J. Kim, "Nonlinear state feedback control of PEM fuel cell systems," in Proc. ICEMS, pp. 1-5, Nov. 2009.

[18] E.-S. Kim, and C.-J. Kim, "Nonlinear observer based control of PEM fuel cell systems," in Proc. INTELEC, pp. 1-3, Oct. 2009.

[19] J. Li, L. Xu, J. Hua, and M. Ouyang, “A distributed real-time control system for PEM fuel cell engine,” in Proc. FUELCELL2009, ASME, pp. 501-507, Jun. 2009.

[20] K. Mammar and A. Chaker, "Fuzzy logic control of fuel cell system for residential power generation,” Journal of Electrical Engineering, Vol. 60, No. 6, pp. 328-334, 2009.

[21] T. Bjažić, "Adaptive control of the DC/DC boost converter supplied by the fuel cell," Ph.D. Thesis, University of Zagreb, Faculty of Electrical Engineering and Computing, Nov. 2010.

[22] *, BCS Fuel Cell Model 64-32 - Operating Manual, BCS Fuel Cells, Inc., 2006.

[23] N. Perić, M. Jelavić, Ž. Ban, H. Domitrović, B. Matijašević, M. Kostelac, and S. Mikac, "Wind turbine control research in the laboratory for renewable energy sources,” in Proc. EWEC, pp. 1-7, Apr. 2010.

[24] T. Bjažić, Ž. Ban, and M. Milanovič, "Modeling of current mode controlled boost converter supplied by fuel cell suitable for controller design purposes," Journal of Power Sources, Vol. 198, pp. 203-217, Jan. 2012.
[25] *, Electronic Load Mainframes: Models 6050A and 6051A - Operating Manual, Agilent Technologies, 2000.

[26] *, 600 W Electronic Load Module: Agilent Model 60504B - Operating Manual, Agilent Technologies, 1991.

[27] *, CompactRIO Developers Guide, National Instruments, 2009.

[28] *, LabVIEW FPGA, National Instruments, 2009.

[29] K. J. Aström, B. Wittenmark, Adaptive Control, Addison-Wesley Publishing Company, Reading, Massachusetts, 1989.

[30] Y. A. Landau, Adaptive Control: The Model Reference Approach, Marcel Dekker, New York, 1979.

[31] M. Farhood, and G. E. Dullerud, "Control of nonstationary LPV systems,” Automatica, Vol. 44, No. 8, pp. 2108-2119, Aug. 2008.

[32] R. Toscano, "Robust synthesis of a PID controller by uncertain multimodel approach," Information Sciences, Vol. 177, No. 6, pp. 1441-1451, Mar. 2007.

[33] Y. A. Bortsov, and N. D. Polyakhov, V. V. Putov, Electromechanical Systems with Adaptive and Modal Control, Energoatomizdat, Leningrad, 1984.

[34] H. Butler, Model Reference Adaptive Control - From Theory to Practice, Prentice Hall, New York, London, 1992.

[35] S. Boyd, Basics on optimization - lecture notes, http://stanford.edu/ boyd/, [Last online access 2011/09/29], 2003.

[36] M. Kvasnica, P. Grieder, M. Baotić, and M. Morari, Multi parametric toolbox (MPT), http://control.ee.ethz.ch/ mpt/, [Last online access 2011/09/29], 2003.

[37] Ž. Ban, Optimization of the model reference adaptive system with parameter adaptation, Ph.D. Thesis, University of Zagreb, Faculty of Electrical Engineering and Computing, 1999.

[38] A. Mujanović, Optimization of the adaptive system with reference model and signal adaptation, Ph.D. Thesis, University of Zagreb, Faculty of Electrical Engineering and Computing, 1997.

[39] *, Matlab - Optimization Toolbox User's Guide, The MathWorks, Inc., Natick, 2009.

[40] T. Bjažić, T. Pavlović, and Ž. Ban, "Estimation of boost converter state-space variables using fast output sampling method,” Journal of Energy and Power Engineering, Vol. 5, No. 1, pp. 88-96, Jan. 2011.

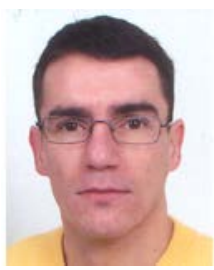

Toni Bjažić (1980) received the B.Sc. and $\mathrm{Ph} . \mathrm{D}$. degrees in electrical engineering from University of Zagreb, Faculty of Electrical Engineering and Computing, Zagreb, Croatia in 2004 and 2010 respectively. Since December 2004, he has been working as a research assistant at the Faculty of Electrical Engineering and Computing, and since December 2010, as a senior research assistant at the same faculty. Since October 2012 he has been working at the Polytechnic of Zagreb as senior lecturer. At the beggining of his employment at the Faculty of Electrical Engineering and Computing he was involved in research on adaptive and optimal control of the 
electrical drives and intelligent and adaptive control of the systems. From 2006 onwards, his research activities include intelligent control of the fuel cell energy sources, as well as control of the microgrid consisted of different renewable energy sources.

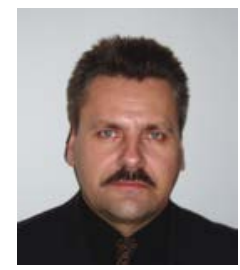

Željko Ban (1962) received B.Sc., M.Sc., and Ph.D. degrees from the University of Zagreb, Faculty of Electrical Engineering and Cobmputing in 1985, 1991, and 1999 respectively. Between 1985 and 1988 he worked as research fellow at Končar Institute of electrical engineering in Zagreb. Since 1988 he has been with the Faculty of Electrical Engineering, University of Zagreb. His current position is the associate professor at Department of Control and Computer Engineering in Automation, Faculty of Electrical Engineering and Computing, University of Zagreb. His research interests were concerned on adaptive and optimal control of the electrical drives and intelligent and adaptive control of the systems. From the 2006 his research activities were concerned on intelligent control of the fuel cell energy sources, as well as control of the microgrid consisted of photovoltaic systems, fuel cell systems and wind energy sources. In his professional work, Ž. Ban was project leader of two projects related to adaptive control and control of the fuel cells energy sources, financed by Ministry of science and technology of Republic of Croatia. Projects were as follows: Intelligent and adaptive control of the systems, and Control system of the fuel cells energy source with the cogeneration. Besides that, Ž. Ban was involved in several projects in cooperation with the Croatian industry related to modelling simulation and controller design of the system. He was also involved in the cooperation with United Technology Research Centre, East Hartford, USA in research problems related to Solar Irradiance, Wind Speed and Wind Turbine Models for the design and control of Hybrid Power Systems. Currently he is involved as a team member in the FP7 ACROSS project.

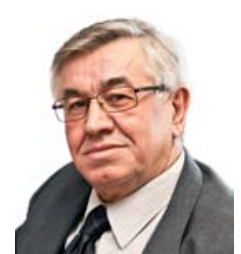

Nedjeljko Perić (1950) has been professionally working for the last thirty five years as a scientist and researcher in the area of automatic control and automation of complex processes and systems. His scientific and professional work can be grouped into two distinct phases. During the first phase he was working at the Končar's Institute of Electrical Engineering (1973-1993) with emphasis on development of the automation systems for complex processes. In particular, he initiated and led the corporate research and development programs for the microprocessor control of electrical machines and related fast processes. The second phase of his work is connected to the employment at the Faculty of Electrical Engineering and Computing in Zagreb (from 1993 onwards), where he has initiated a broad research activity in advanced control of complex and large scale technical systems. His research results are published in scientific journals (more than 30 papers), proceedings of international conferences (more than 200 papers), and in numerous research studies/reports (more than 60 reports). Prof. Peric has particularly excelled in the leadership of national and international research projects. For his work he has received numerous awards, among others, the Croatian national award for science (for year 2007) for important scientific achievement in development of advanced control and estimation strategies for complex technical systems. He has also received the "Fran Bošnjaković" award (in 2009) for the exceptional contribution to the development and promotion of the automatic control research field within the area of technical sciences. Currently, he holds the post of the Dean of the Faculty of Electrical Engineering and Computing, University of Zagreb. 